\title{
The quark-gluon plasma and D6-branes on the conifold
}

\author{
Johannes Schmude* \\ Department of Physics \\ Swansea University, Swansea, SA2 8PP, United Kingdom
}

\begin{abstract}
We investigate the possibility of constructing a supergravity background dual to the quark-gluon plasma using D6-branes wrapping a threecycle in the deformed conifold. The UV-completion of this setup is given by M-theory on a $G_{2}$ holonomy manifold. For the class of metrics considered we find that there are only non-extremal D-brane solutions in the limit of the singular conifold with the singularity being resolved by the D-brane horizon. The thermodynamic properties of the system show some puzzling features, such as negative specific heat at an unusual behavior of the entropy. Among the properties of the plasma studied using this holographic dual are the quark-antiquark potential, the shear viscosity and parton energy loss. While one finds the expected behavior for the potential and the viscosity - deconfinement and the universal shear-viscosity to entropy ratio - both the jet quenching parameter and the calculation of the drag force lead us to the conclusion that there is no parton energy loss in the dual plasma. Our results indicate that the background constructed is not dual to a realistic QGP, yet we argue that this should improve upon inclusion of the three-form gauge potential in the elevendimensional background.
\end{abstract}

*pyjs@swansea.ac.uk 


\section{Contents}

1 Introduction \& Summary 2

2 The supergravity dual at zero temperature $\quad 4$

2.1 M-theory on the $G_{2}$ holonomy manifold . . . . . . . . . . . . . . 4

2.2 Wrapped D6-branes in type IIA string theory . . . . . . . . . 5

3 The gauge theory at zero temperature $\quad \mathbf{8}$

3.1 The coupling constant of the gauge theory . . . . . . . . . 8

3.2 Field Content . . . . . . . . . . . . . . . . . . 10

3.3 The gauge/gravity correspondence . . . . . . . . . . . . . . 10

3.4 Wilson loops and minimal surfaces . . . . . . . . . . . . . . . 12

3.4.1 Boundary conditions ................. 13

3.5 The quark-antiquark potential \& confinement . . . . . . . . . . . 14

4 The supergravity theory at finite temperature 18

4.1 The eleven-dimensional black hole . . . . . . . . . . . . 18

4.2 Thermodynamics . . . . . . . . . . . . . . . . . 19

4.3 Comparison with the Schwarzschild solution . . . . . . . . . . 23

4.4 Dimensional Reduction . . . . . . . . . . . . . . . . . 24

5 The field theory at finite temperature 24

5.1 Properties of the Dual Field Theory . . . . . . . . . . . . . . 24

5.2 Quark-Antiquark Potential ............. . . 25

5.3 Shear Viscosity . . . . . . . . . . . . . . . . . 26

5.4 Energy Loss of a Heavy Quark . . . . . . . . . . . . . . . . 27

5.4.1 Experimental Background . . . . . . . . . . . . . . 27

5.4 .2 Jet Quenching . . . . . . . . . . . . . . . . . . 28

5.4 Drag Force ................... . . 33

6 Conclusions $\quad 35$

A The bundle structure of $S^{3} \quad 36$

\section{Introduction \& Summary}

As it is well known, the gauge/string theory correspondence relates strongly coupled gauge- with weakly coupled string theories and vice versa $[1,2,3]$. Further developments in the field lead to studies of the non-perturbative quarkgluon plasma (QGP) as produced in relativistic heavy ion collisions $[4,5,6,7]$ or as relevant to the physics of the early universe and super dense stars. Among the items that were studied using a gravity dual are the plasma's shear viscosity [8], photoproduction [9], jet-quenching [10], and drag force [11]. ${ }^{1}$

A large portion of the research conducted in this area centers on $\mathcal{N}=4$ super Yang-Mills and AdS/CFT in its best understood form, D3-branes in type IIB theory. Apart from the fact that this is the most tractable of gravity duals, one reason for choosing $\mathcal{N}=4$ is that albeit having properties very different

\footnotetext{
${ }^{1} \mathrm{~A}$ recent review on the uses of gauge/string duality and QGP physics is [12]. The general properties of the plasma in general and RHIC physics are summarized in [13] and [14].
} 
from those of QCD at $T=0$, the two theories start to appear more and more similiar as soon as there is finite temperature. Despite these successes however a complete study of QGP physics based on string theory demands for an investigation of the $T \neq 0$ behavior of other gravity duals showing a stronger resemblance to QCD even at zero temperature. Some work in this direction was undertaken in $[15,16,17,18,19]$

In this paper we investigate the possibility of constructing a supergravity background dual to an $\mathcal{N}=1$ QGP based on D6-branes wrapping an $S^{3}$ in the deformed conifold. In order for some supersymmetry to be preserved, the field theory living on the world-volume of the branes has to be topologically twisted [20]. Apart from the usual gauge/gravity correspondence, the theory exhibits a further large $N$ duality, the conifold transition, whose history starts with [21]. Here it was shown that topological string theory on a blown up CalabiYau conifold is equivalent to Chern-Simmons gauge theory on $S^{3}$ at large $N$. This duality reappears in the context of the AdS/CFT correspondence when considering $N$ D6-branes wrapping an $S^{3}$ in the deformed conifold [22], as the conifold transition connects this setup to type IIA string theory on the resolved conifold without any branes but with $N$ units of Ramond-Ramond flux through an $S^{2}$. Independently of whether one starts from the resolved or the deformed conifold, when lifting to M-theory the geometry is that of the spin bundle over $S^{3}$, a manifold with $G_{2}$ holonomy [23], and the duality takes the form of the flop transition [24]. The connection to 8-dimensional gauged supergravity was established in [25].

The duality resurfaces in the gauge-theory as follows. For $\lambda=N g_{Y M}^{2}=$ $N g_{s} \ll 1$, the gauge-theory is best described by the $N$ D6-branes wrapping the $S^{3}$. For large 't Hooft coupling however, one needs to consider the branes' gravitational backreaction and makes therefore use of the resolved conifold. The theory is pure $\mathcal{N}=1$ super Yang-Mills with additional massive degrees of freedom from Kaluza-Klein reduction. We will see it is not possible to fully decouple these modes. Also, as was already shown in [26] for the case of flat D6-branes, one cannot expect the gauge theory to fully decouple from gravity.

If one wants to use this gravity dual to study the QGP, one needs to add a black hole to the supergravity background. As the theory is purely gravitational when lifting to eleven dimensions, the equations of motion take the simplest form possible here,

$$
R_{\mu \nu}=0,
$$

making this the best place to perform the search for a black hole solution. As we find in section 4 , if one wants to keep the ansatz for the new metric as simple as possible by making the substitutions

$$
d t^{2} \rightarrow f(\rho) d t^{2} \quad d \rho^{2} \rightarrow \frac{d \rho^{2}}{f(\rho)},
$$

there is a non-trivial solution if and only if one makes the geometry of the $G_{2}$ manifold singular. The unique solution is then $f=1-\rho_{h}^{5} / \rho^{5}$, where the singularity at $\rho=0$ is hidden by the horizon $\rho_{h}>0$. When studying the thermodynamics of this new solution, we will see that the black hole behaves in many ways as the ordinary Schwarzschild solutions in four and eleven dimensions. I.e. the temperature is proportional to the inverse of the horizon, $T=\frac{5}{4 \pi \rho_{h}}$, and the specific heat is negative. As the horizon of the black hole 
covers the six-dimensional base of the internal $G_{2}$ cone, the entropy behaves as $S \propto \rho_{h}^{6}$, leading to the surprising relation $S \propto T^{-6}$. While our subsequent calculation of the quark-antiquark potential and the shear-viscosity show the expected results, that is confinement and a shear-viscosity to entropy ratio of $\eta / s=1 / 4 \pi$, the discussion of parton energy loss leads to a puzzling pathological property of the solution. The energy loss as calculated from the jet-quenching parameter and the damping coefficient of the drag force are both vanishing.

The organization of the paper is as follows. Sections 2 and 3 are dedicated to an extensive review of the string theory and its gauge dual at zero temperature. Here our discussion starts with eleven-dimensional supergravity and then proceeds via type IIA to the four-dimensional super Yang-Mills theory. As we will make extensive use of the machinery of Wilson lines, we shall give a brief introduction to this subject before calculating the quark-antiquark potential, paying special attention to the boundary conditions imposed on worldsheets used to calculate Wilson lines. After these preliminaries we finally turn to the subject of finite temperature. The discussion mimicks that of the $T=0$ case in that we will start from the eleven-dimensional gravity dual (section 4) and then progress via type IIA to the gauge-theory (section 5). Here we study the quark-antiquark potential, the shear-viscosity, and parton energy loss as it is parametrized by the jet-quenching factor $\hat{q}$ and the drag-force. The conclusions in section 6 are followed with an appendix reviewing the bundle structure of the three-sphere (appendix A).

As we mentioned earlier, sections 2,3 and A contain mostly review material. ${ }^{2}$ The reader familiar with the items discussed here might therefore prefer to start with section 4 referring to the others when necessary.

\section{The supergravity dual at zero temperature}

We begin with a review of the supergravity dual of the zero temperature theory. Depending on the energy scale of of the processes one would like to study this is either eleven-dimensional M- or ten-dimensional type IIA string theory. We follow a top-down approach, starting with the UV regime given by M-theory and ignoring the properties of the dual field theory until section 3 .

\section{$2.1 \quad$ M-theory on the $G_{2}$ holonomy manifold}

We will see shortly that the gauge-theory we are interested in is living on the world volume of $N$ D6-branes wrapping a calibrated three-cycle in the deformed conifold. As mentioned before, the UV completion of this theory is given by M-theory on the spin bundle over $S^{3}$, a manifold with $G_{2}$ holonomy. This setup was discussed in [24, 25]. This background is purely gravitational (i.e. all fields except the metric are set to zero) and given by the metric

$$
d s_{M}^{2}=d x_{1,3}^{2}+\frac{d \rho^{2}}{1-a^{3} / \rho^{3}}+\frac{\rho^{2}}{12} \tilde{w}^{a 2}+\frac{\rho^{2}}{9}\left(1-\frac{a^{3}}{\rho^{3}}\right)\left(w^{a}-\frac{1}{2} \tilde{w}^{a}\right)^{2} .
$$

Ignoring the four dimensions of Minkowski space, $\mathbb{R}^{3,1}$, this has asymptotically the structure of a cone with base $\tilde{S}^{3} \times S^{3}$. Each sphere is parametrized by a set

\footnotetext{
${ }^{2}$ However the discussion of super Yang-Mills coupling constant in section 3.1 and the $q \bar{q}-$ potential in section 3.5 have not been published in the literature so far.
} 
of one-forms $w^{a}$, whose explicit form is

$$
\begin{aligned}
& w^{1}=\cos \phi d \theta+\sin \theta \sin \phi d \psi \\
& w^{2}=\sin \phi d \theta-\sin \theta \cos \phi d \psi \\
& w^{3}=d \phi+\cos \theta d \psi,
\end{aligned}
$$

with

$$
\theta \in[0, \pi] \quad \phi \in[0,2 \pi] \quad \psi \in[0,4 \pi] .
$$

Also note that $\rho \in[a, \infty)$. $d x_{1,3}^{2}$ denotes the usual metric on $\mathbb{R}^{1,3}$ with mostly positive signature.

A look at the metric tells us that in opposite to $S^{3}, \tilde{S}^{3}$ has a finite radius $a$ as we take $\rho \rightarrow a$. This resolves the singularity at $\rho=a$. Naturally one could also have picked the other sphere, $S^{3}$, to do this. Defining the volume of $S^{3}$ in this case to be $-a$ with $a<0$, it appears as if the moduli space of M-theory on this space is given by $a \in \mathbb{R} \backslash\{0\}$. I.e. the moduli space decomposes into two disconnected components. If we wanted to move from one sector to the other, we'd have to pass through the singularity. However, as Atiyah, Maldacena, and Vafa showed [24], it is indeed possible to continuously pass from one component to the other. The point is that M-theory contains also a three-form potential $C_{(3)}$, and turning on a $C_{(3)}$ flux through one of the spheres smoothens out the singularity. Denoting the flux by $C$ and writing $a+\imath \int_{S^{3}} C$, they showed that the true moduli space is the punctured complex plane consisting of a single path component. The resulting duality is known as the flop transition.

As mentioned, the metric $(2.1)$ has $G_{2}$ holonomy. Being a subgroup of $\mathcal{S O}(7), G_{2}$ may be embedded into $\operatorname{Spin}(7)$. The spinor representation of the latter is an $\mathbf{8}$, which decomposes for the $G_{2}$ subgroup as $\mathbf{7} \otimes \mathbf{1}$. Transporting any spinor $\Psi$ around a closed loop, it transforms as

$$
\Psi \mapsto d_{g} \Psi \quad g \in G_{2},
$$

where $d_{g}$ denotes a suitable representation. Only the singlet is invariant under this operation. Discarding all those spinor fields transforming unde the $\mathbf{7}$ leaves us with $\frac{1}{8}$ of the original supersymmetry. As we started with 32 supercharges, we are now dealing with a theory with 4 supercharges.

\subsection{Wrapped D6-branes in type IIA string theory}

A brief look at appendix A tells us that $S^{3}$ is a $\mathcal{U}(1)$ principal bundle over $S^{2}$. Thus when flowing towards the IR regime, and the size of the eleventh direction decreases, it is natural to perform a dimensional reduction along one of the two $S^{1}$ s leading to an effective description in terms of type IIA string theory on a space with topology

$$
\mathbb{R}^{1,3} \times \mathbb{R}_{+} \times S^{3} \times S^{2}
$$

If we choose an $S^{1}$ in the singular three-sphere, $S^{1} \subset S^{3}$, the resulting geometry is a singular $S^{2}$ and a non-singular $S^{3}$ known as the deformed conifold. See fig. 1(a). The converse case, the resolved conifold, is depicted in fig. 1(c). In general the conifold, as discussed in [27], is a six-dimensional non-compact manifold which is a cone with base $S^{3} \times S^{2}$. As depicted in fig. 1(b), there is a singularity at which both spheres have a vanishing radius. From a mathematician's point of view one deals with this singularity by giving one of the spheres a 


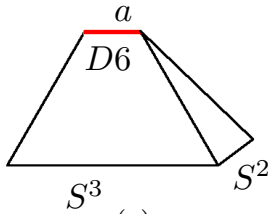

(a)

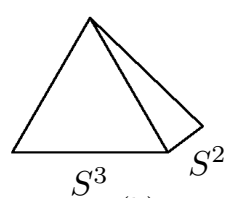

(b)

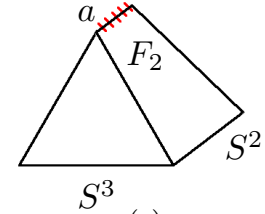

(c)

Figure 1: The deformed 1(a), singular 1(b), and resolved 1(c) conifold. In the type IIA theory discussed in section 2.2 , there are $N$ D6-branes wrapping the non-vanishing $S^{3}$ in 1(a), while in in the dual geometry 1 (c) the branes have disappeared and been replaced by a two-form flux $F_{2}$.

finite radius, leading to the deformed and the resolved conifold. Physics allows for the following interpretation of this ${ }^{3}[24,28]$ : If one considers the singularity as the $a \rightarrow 0$ limit of the deformed conifold, there is a logarithmic singularity in the metric. This may be interpreted as the result of having integrated out a field whose mass is dependent on $a, m=m(a)$. When approaching the singularity,

$$
m(a) \rightarrow 0 \quad \text { as } \quad a \rightarrow 0 .
$$

Therefore the physical interpretation of the singularity lies in the fact that one has attempted to integrate out a massless field. As we will see in section 4.1 however, the finite temperature theory makes use of another method of dealing with the singularity. The theory will be defined on the singular conifold with the singularity hidden behind a black hole's event horizon.

The string theory equivalent of the flop transition is the conifold transition[22]. It relates the two geometries via a large $N$ duality. For small 't Hooft coupling

$$
\lambda=N g_{Y M}^{2}=N g_{s} \ll 1,
$$

one considers a stack of $N$ D6-branes wrapping the non-singular $S^{3}$ in the deformed conifold. Taking the 't Hooft coupling large on the other hand one cannot neglect the branes' backreaction and does therefore pass to the resolved conifold. Here the branes have disappeared and been replaced by $N$ units of two-form flux through the now blown up $S^{2}$.

Being interested in a strongly coupled quark-gluon plasma, we choose to reduce along the non-singular $S^{1} \subset \tilde{S}^{3}$. Before doing so, we have to identify the $S^{1}$ fibre along which we want to reduce. A generic three-sphere may be written as

$$
S^{3} \equiv\left\{\left.\left(z_{0}, z_{1}\right) \in \mathbb{C}^{2}|| z_{0}\right|^{2}+\left|z_{1}\right|^{2}=1\right\} .
$$

The coordinates $z_{0,1}$ are related to those of $(2.2)$ by

$$
\begin{aligned}
& z_{0}=\cos \frac{\tilde{\theta}}{2} e^{\imath \frac{\tilde{\psi}+\tilde{\phi}}{2}} \\
& z_{1}=\imath \sin \frac{\tilde{\theta}}{2} e^{\imath \frac{\tilde{\psi}-\tilde{\phi}}{2}} .
\end{aligned}
$$

\footnotetext{
${ }^{3}$ The interpretation of the singularity in terms of having integrated out a massless field appears in [28] for the generic case of string theory on a deformed conifold. We did not explicitly verify that it holds in our case.
} 
(A.3) tells us, that the projection $S^{3} \stackrel{\pi}{\rightarrow} S^{2}$ acts on this as

$$
\begin{array}{ll}
-\imath \cot \frac{\tilde{\theta}}{2} e^{\imath 2 \tilde{\phi}} & \tilde{\theta} \neq 0 \\
\imath \tan \frac{\tilde{\theta}}{2} e^{-\imath 2 \tilde{\phi}} & \tilde{\theta} \neq \pi,
\end{array}
$$

depending on the coordinate patch. One sees immediately that the fibre coordinate is $\tilde{\psi}$, as it does not survive the projection.

Before actually reducing we mod out by

$$
\mathbb{Z}_{N} \subset S^{1} \subset \tilde{S}^{3} .
$$

This means a change in the periodicity of $\tilde{\psi}$,

$$
\begin{aligned}
\tilde{\psi} \in[0,2 \pi] & \rightarrow \tilde{\psi} \in[0,2 \pi / N], \\
d \tilde{\psi} & \rightarrow \frac{d \tilde{\psi}}{N}
\end{aligned}
$$

As we will see soon, $N$ gives the $F_{2}$ flux through $\tilde{S}^{2}$ and therefore the number of D6-branes present in the dual type IIA geometry.

In order to perform the reduction, we could simply expand the metric. However, there is a smarter way to go about this. Defining

$$
n^{a} \equiv \tilde{w}^{a}\left(\partial_{\tilde{\psi}}\right)=(\sin \tilde{\theta} \sin \tilde{\phi},-\sin \tilde{\theta} \cos \tilde{\phi}, \cos \tilde{\theta})
$$

we may rewrite the metric (2.1) in terms of a new set of differential forms $\hat{w}^{a}$ independent of $d \tilde{\psi}$,

$$
\tilde{w}^{a}=\hat{w}^{a}+n^{a} \frac{d \tilde{\psi}}{N} .
$$

Writing $\beta=1-a^{3} / \rho^{3}$, we obtain

$$
\begin{aligned}
d s_{\mathrm{M}}^{2} & =d x_{1,3}^{2}+\frac{d \rho^{2}}{\beta}+\frac{\rho^{2}(3+\beta)}{36} \hat{w}^{2}+\frac{\rho^{2}}{9} \beta w^{2}-\frac{\rho^{2}}{9} \beta w \cdot \hat{w} \\
& +\underbrace{\frac{\rho^{2}(3+\beta)}{36 N^{2} a^{2}}}_{e^{\frac{4 \Phi}{3}}} a^{2} d \tilde{\psi}^{2}+\underbrace{\left(\frac{2 \rho^{2}}{12 N a} n \cdot \hat{w}+\frac{\rho^{2}}{18 N a} \beta n \cdot \hat{w}-\frac{\rho^{2}}{9 N a} \beta n \cdot w\right)}_{2 e^{\frac{4 \Phi}{3}} A_{(1)}} a d \tilde{\psi}
\end{aligned}
$$

We included several factors of $a$ to make sure that everything has the correct dimensions. Dimensional reduction along an $S^{1}$ yields apart from the new metric $g_{\mu \nu}$ a one-form potential and the dilaton. We may read them of from the elevendimensional metric $G_{M N}$ using [29]

$$
G_{M N}=e^{-\frac{2}{3} \Phi}\left(\begin{array}{cc}
g_{\mu \nu}+A_{\mu} A_{\nu} e^{2 \Phi} & A_{\mu} e^{2 \Phi} \\
A_{\nu} e^{2 \Phi} & e^{2 \Phi}
\end{array}\right)
$$


Thus

$$
\begin{aligned}
e^{\frac{4 \Phi}{3}} & =\frac{\rho^{2}(3+\beta)}{36 N^{2} a^{2}} \\
A_{(1)} & =N a\left(\hat{w} \cdot n-\frac{2 \beta}{3+\beta} w \cdot n\right) \\
d s_{I I A}^{2} & =e^{\frac{2}{3} \Phi}\left(d x_{1,3}^{2}+\frac{d \rho^{2}}{\beta}+\frac{\rho^{2}(3+\beta)}{36} \hat{w}^{2}+\frac{\rho^{2}}{9} \beta w^{2}\right. \\
& \left.-\frac{\rho^{2}}{9} \beta w \cdot \hat{w}-e^{\frac{4}{3} \Phi} A_{(1)} A_{(1)}\right) .
\end{aligned}
$$

We will also need the ten-dimensional Ricci scalar. In the string frame it reads

$$
R=-9 a N \frac{832 \rho^{9}-240 a^{3} \rho^{6}+63 a^{6} \rho^{3}-7 a^{9}}{2 \sqrt{4-\frac{a^{3}}{\rho^{3}}} \rho^{6}\left(4 \rho^{3}-a^{3}\right)^{2}} .
$$

$R$ is not singular at $\rho=a$. As a matter of fact,

$$
\left.R\right|_{\rho=a}=-108 \sqrt{3} \frac{N}{a^{2}}
$$

which gives us an explicit expression for the conifold singularity in the limit $a \rightarrow 0$.

We claimed that in the above geometry there are $N$ units of RamondRamond flux through the two-sphere. To check this we simply calculate the $F_{(2)}$ flux through the $S^{2}$ parametrized by $\tilde{\theta}$ and $\tilde{\phi}$.

$$
\int_{S^{2}} * F_{(8)}=\int_{S^{2}} * * F_{(2)}=-\int_{S^{2}} d A_{(1)}=4 \pi N a
$$

Now the conifold transition relates the above to a stack of $N$ D6-branes on the deformed conifold. One may obtain this dual geometry from elevendimensional supergravity by reducing along the singular three-sphere. Indications towards the presence of the branes are the resulting one-form potential, which couples magnetically to the branes, and the behavior of the Ricci scalar near the singularity. See [25, 23].

\section{The gauge theory at zero temperature}

We shall now turn to the discussion of the dual gauge theory at $T=0$. With the exception of the Yang-Mills coupling in section 3.1 and the $q \bar{q}$-potential in section 3.5 this section contains mostly review material. The relation between the supergravity backgrounds, the gauge theory, and gauged supergravity was exhibited in [25]. For a review on this issue see [30].

\subsection{The coupling constant of the gauge theory}

In the following we elaborate on the developments in $[31,32]$. To find the super Yang-Mills theory's coupling constant $g_{Y M}$, we place a D6-probe brane at constant $\rho$, extending along $x^{\mu}$ and wrapping the resolved conifold's $S^{3}$. Recall 
that we may think of our original stack of D6-branes as wrapping $\tilde{S}^{3}$ in the deformed conifold. We also fix the brane's position in the $S^{2}$ to be $\tilde{\theta}=\tilde{\phi}=0$. The general idea is to identify the gauge field living on the probe brane with that of the dual super Yang-Mills theory. Thus we may extract information about the dual theory from the probe's DBI action. Using world-volume coordinates $\xi^{a}$ and labeling the brane-tension $T_{6}$, we expand the DBI action in powers of $\alpha^{\prime}$

$$
\begin{aligned}
S_{D B I} & =-T_{6} \int d^{7} \xi e^{-\Phi} \sqrt{-\operatorname{det} \mathcal{P}[g]+2 \pi \alpha^{\prime} F}+T_{6} \int \sum_{n} C_{(n)} \wedge e^{2 \pi F} \\
& =-T_{6} \int d^{7} \xi e^{-\Phi} \sqrt{-\operatorname{det} \mathcal{P}[g]}\left(1+\left(\alpha^{\prime} \pi\right)^{2} F^{2}\right)+\mathcal{O}\left(\alpha^{\prime}\right)^{3}+\ldots
\end{aligned}
$$

$\mathcal{P}$ denotes the pullback onto the brane. For the embedding we have chosen, the induced metric $\mathcal{P}[g]$ is

$$
d s_{6}^{2}=e^{\frac{2}{3} \Phi}\left(d x_{1,3}^{2}+\frac{\rho^{2}}{9} \beta w^{2}-e^{\frac{4}{3} \Phi} N^{2} a^{2}\left(\frac{2 \beta}{3+\beta}\right)^{2}\left(w^{3}\right)^{2}\right)
$$

Now notice that after Kaluza-Klein decomposition the massless modes of $F_{\mu \nu}$ are functions of the $x^{\mu}$ alone, while all the other terms in (3.1) do not depend on the flat part of the world-volume. Therefore that part of (3.1) containing $F^{2}$ may be written as

$$
-\left(T_{6}\left(\pi \alpha^{\prime}\right)^{2} \int d \theta d \phi d \psi e^{-\Phi} \sqrt{-\operatorname{det} \mathcal{P}[g]}\right) \int d^{4} x F^{2} .
$$

Comparing the Yang-Mills action

$$
S_{Y M}=-\frac{1}{4 g_{Y M}^{2}} \int d^{4} x F^{2}+\frac{\theta_{Y M}}{32 \pi^{2}} \int d^{4} x F \tilde{F}
$$

and using the explicit expression for the D-brane tension

$$
T_{p}=\frac{1}{(2 \pi)^{p} \alpha^{\prime \frac{p+1}{2}}}
$$

we obtain

$$
g_{Y M}=18(12)^{\frac{1}{4}} \frac{N a \pi \sqrt{\alpha^{\prime 9 / 2} \rho}}{\left(\left(4 \rho^{3}-a^{3}\right)\left(\rho^{3}-a^{3}\right)^{3}\right)^{1 / 4}} .
$$

Note that the coupling is dimensionless, as it should be the case for a fourdimensional Yang-Mills theory. We have plotted $g_{Y M}$ in figure 2. The AdS/CFT dictionary tells us that we may relate the radial coordinate $\rho$ to the energy scale. To obtain a precise relation one may consider chiral symmetry breaking and the vev of the gluino condensate $\langle\lambda \lambda\rangle$ [31]. Yet for our purposes it is sufficient to think of $\rho \rightarrow \infty$ as the UV regime of the gauge theory and $\rho \rightarrow a$ as the IR. ${ }^{4}$ Then (3.6) clearly shows asymptotic freedom.

\footnotetext{
${ }^{4}$ As we mentioned earlier, the UV completion is given by M-theory, while in the infrared the relevant degrees of freedom are best described by the gauge theory. See section 3.3 and [26].
} 


\subsection{Field Content}

We shall take a look at the massless excitations. Prior to wrapping, the theory living on the world volume of $N$ D6-branes is a super Yang-Mills theory with 16 supercharges, as the branes are half-BPS. Upon wrapping, the global symmetries break as

$$
\mathcal{S O}(1,6) \times \mathcal{S O}_{R}(3) \rightarrow \mathcal{S O}(1,3) \times \mathcal{S O}(3) \times \mathcal{S O}_{R}(3)
$$

From dimensional analysis it follows that the Kaluza-Klein modes become relevant at energy scales of order

$$
\Lambda_{\mathrm{KK}} \sim \frac{\alpha^{\prime 3 / 2}}{\operatorname{Vol} S^{3}}=\frac{\alpha^{\prime 3 / 2}}{2 \pi^{2} a^{3}} .
$$

Ignoring all massive modes, the bosonic sector includes now the gauge potential and three massless scalars transforming as a $\mathbf{3}$ under the R-symmetry. The representation for the fermions changes under $(3.7)$ from $(\mathbf{8}, \mathbf{2})$ to $(\mathbf{4}, \mathbf{2}, \mathbf{2})$.

This is not the complete picture however. Consider the behavior of the gravitino under SUSY transformations,

$$
\left.\delta_{\epsilon} \Psi_{\mu}\right|_{\Psi=0}=\nabla_{\mu} \epsilon=\left(\partial_{\mu}+\frac{1}{2} \omega_{\mu}\right) \epsilon
$$

with $\omega$ being the spin connection. For the theory to be supersymmetric we need a covarianntly constant spinor satisfying $\nabla_{\mu} \epsilon=0$. As the spin structure of $S^{3}$ does not allow for such a spinor to exist, supersymmetry is completely broken upon wrapping. Raising the status of the R-symmetry to that of a gauge symmetry, we may modify (3.9) to

$$
\nabla_{\mu} \epsilon=\left(\partial_{\mu}+\frac{1}{2} \omega_{\mu}+A_{\mu}^{(R)}\right) \epsilon .
$$

Fixing $A_{\mu}^{(R)}=2 \omega_{\mu}$ resolves the issue. This topological twist was first introduced by Witten in [20]. While it changes the behavior of the $6+1$ dimensional theory significantly, the consequence for the $3+1$ dimensional one we are interested in consists in keeping only those fields that transform as a singlet under the diagonal

$$
\mathcal{S O}(3) \times \mathcal{S O}_{R}(3) \rightarrow \mathcal{S O}_{D}(3)
$$

The gauge potential is not affected by the whole construction, whereas all of the scalars disappear from the spectrum. The representation of the fermions decomposes as

$$
(4,2,2) \rightarrow(4,1) \oplus(4,3),
$$

because $\mathbf{2} \times \mathbf{2}=\mathbf{1} \oplus \mathbf{3}$. So recalling that the branes are half-BPS we are left with $\frac{1}{2} \times \frac{1}{4} \times 32=4$ supercharges, confirming the previous calculation based on the holonomy of the eleven-dimensional background. Thus the massless spectrum is given by pure $\mathcal{N}=1$ super Yang-Mills theory.

\subsection{The gauge/gravity correspondence}

Knowing the energy scale of the KK-modes (3.8) and the behavior of the Ricci scalar (2.20), the Yang-Mills coupling constant (3.6), and the dilaton (2.17) 


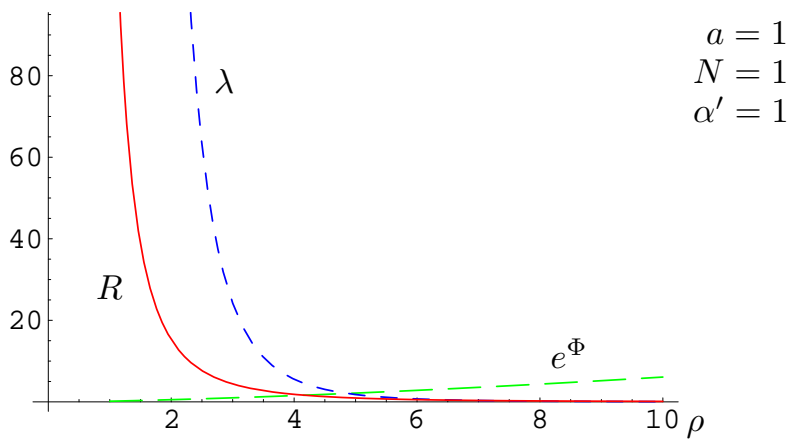

Figure 2: Ricci scalar, 't Hooft coupling, and dilaton in terms of $\rho$. One sees clearly that the IR phsics is captured by type IIA string theory while the UV completion is given by M-theory on the $G_{2}$ holonomy manifold. Note that albeit appearances $R$ is not singular at $\rho=a$. The 't Hooft coupling however is.

enables us to address the issue at which energy scales the system is best described by super Yang-Mills, type IIA, or M-theory. As in the previous section we do not know the precise relation between the radial coordinate $\rho$ and the energy scale $\mu$ in question, and are therefore only able to make qualitative statements identifying the large- $\rho$ regime as the UV and vice versa. Figure 2 shows the behavior of all three relevant quantities.

We see that in the IR the relevant degrees of freedom are best described in type IIA theory. While it might seem that the UV completion is given by both M-theory and super Yang-Mills one should not forget that figure 2 shows the four-dimensional gauge coupling. At sufficiently high energies the $\mathcal{N}=1$ theory will begin to fully explore the compact dimensions; the gauge theory becomes $6+1$ dimensional. Purely gravitational M-theory gives the only UV-completion.

If we want to use this overall setup to study zero-temperature, non-perturbative gauge dynamics, it follows from (3.8) that we want the resolution parameter $a$ to satisfy $a<\sqrt{\alpha^{\prime}}$. However we also need

$$
\alpha^{\prime} R \ll 1 \quad \lambda=g_{Y M}^{2} N>1 \quad e^{\Phi} \ll 1 .
$$

For $\rho \rightarrow a$, these quantities behave as

$$
\begin{aligned}
\lambda & \sim \frac{N^{3} a^{3 / 2} \alpha^{\prime 3 / 2}}{\left(\rho^{3}-a^{3}\right)^{3 / 2}} \\
-\alpha^{\prime} R & \leq \sqrt{3} 108 \frac{N \alpha^{\prime}}{a^{2}} \\
e^{\Phi} & =\frac{1}{\sqrt{2} N^{3 / 2}}\left(\frac{1}{23^{3 / 4}}+\frac{3^{5 / 4}}{8 a}(\rho-a)\right)+\mathcal{O}(\rho-a)^{2}
\end{aligned}
$$

Comparing this with figure 2 we conclude that there is a limit for $N, a, \alpha^{\prime}$ in which the supergravity approximation captures non-perturbative gauge dynamics. However the massive Kaluza-Klein modes do not fully decouple and thus spoil the behavior of pure $\mathcal{N}=1$ super Yang-Mills. If one were able to perform computations beyond the supergravity limit one could easily avoid this issue. 


\subsection{Wilson loops and minimal surfaces}

The AdS/CFT-correspondence is a powerful tool for the study of Wilson lines [33], [34], and [35]. In the next section (3.5) we shall use it to study the $q \bar{q}$ potential at $T=0$. Further applications will be the finite-temperature $q \bar{q}$ potential and the jet-quenching factor in sections 5.2 and 5.4.2 respectively, while the the method used to compute the drag-force in section 5.4.3 takes a similiar approach.

For a generic gauge theory a Wilson loop is defined as ${ }^{5}$

$$
W(\mathcal{C})=\mathcal{P} e^{\imath \oint_{\mathcal{C}} d A} .
$$

$\mathcal{P}$ denotes path ordering and $\mathcal{C}$ the contour of integration.

To see how to calculate the expectation value $\langle W(\mathcal{C})\rangle$ for a generic contour $\mathcal{C}$ using the AdS/CFT-correspondence, consider the following. If we do not close the loop $\mathcal{C}$, but instead consider a line, (3.16) is a non-local operator transforming at it's endpoints under the fundamental- and anti-fundamental representation respectively. The gauge theory and its gravity dual as discussed above are free of any fundamental degrees of freedom. In order to introduce these we start with a stack of $N+1$ D6-branes and place one of them at a large yet finite radius $\rho_{\Lambda}$. The gauge symmetry is broken as

$$
\mathcal{S U}(N+1) \rightarrow \mathcal{S U}(N) \times \mathcal{U}(1) .
$$

We have Higgsed the theory. From the point of view of the gauge theory we therefore expect the appearance of massive W-bosons, which we will treat as highly massive probe quarks. In the string theory these bosons are realized by strings stretching between the stack of branes and the separated one transforming in the (anti-)fundamental representation of the two new gauge groups. The new $\mathcal{U}(1)$ gauge field may be ignored as it's living on the brane which is at a large separation from the stack of D6s. ${ }^{6}$ When taking the decoupling limit the $N$ branes at $\rho=0$ are replaced by the background geometry while the single brane at $\rho_{\Lambda}$ may be treated as a probe. As the branes are replaced by their geometry, the correct way for the $\mathrm{W}$-bosons to interact with the gauge theory is not by ending on the branes but by interacting with the background. Therefore one evaluates $\langle W(\mathcal{C})\rangle$ by embedding the contour $\mathcal{C}$ into the probe brane and using it as a boundary condition for the worldsheets of open-strings exploring the bulk. See figure 3(a). The AdS/CFT-dictionary tells us then to calculate the expectation value of the Wilson loop for the adjoint representation by minimizing the Nambu-Goto action for the corresponding world-sheets,

$$
\left\langle W^{A}(\mathcal{C})\right\rangle=\lim e^{-\mathcal{S}_{N G}} .
$$

\footnotetext{
${ }^{5}$ The expression presented here is not entirely generic. E.g. for $d=4, \mathcal{N}=4$ super Yang Mills whose gravity dual is defined on $A d S_{5} \times S^{5}$, one needs also to consider scalar fields $\Phi^{I}$. The index $I$ may be considered as a representation index of $\mathcal{S O}(6)$. The Wilson line is given by

$$
W^{A}(\mathcal{C})=\mathcal{P} e^{\imath \oint_{\mathcal{C}} d s\left(x^{\mu} A_{\mu}+|\dot{x}| n^{I} \Phi^{I}\right)} .
$$

However, as (3.16) is entirely sufficient in the context presented here, we shall not elaborate on the issue.

${ }^{6}$ An alternative approach would be to take the flavor brane to wrap the $S^{2}$ and to extend along $\rho$ from $\rho_{\Lambda}$ to $\infty$. In this case one argues that the gauge-theory living on the probe is non-dynamical as seen from the four-dimensional theory as the probe wraps a non-compact dimension.
} 


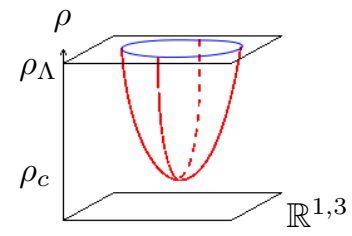

(a)

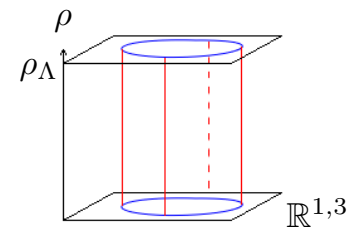

(b)

Figure 3: A Wilson 3(a) loop in the gauge theory is evaluated by using the loop as the boundary condition of a worldsheet ending on a probe brane. The worldsheet reaches a minimum at $\rho=\rho_{c} \geq 0$. The action is renormalised by that of strings stretching straight from the loop to the bottom of the space, sometimes given by the horizon of a black hole 3(b). As was argued in [48], one also needs to consider strings stretching from the probe away from the horizon.

$\mathcal{S}_{N G}$ is the Nambu-Goto action

$$
\begin{aligned}
S_{\mathrm{NG}} & =\frac{1}{2 \pi \alpha^{\prime}} \int d \tau d \sigma \sqrt{-\operatorname{det} \partial_{\alpha} X^{\mu} \partial_{\beta} X_{\mu}} \\
& =\frac{1}{2 \pi \alpha^{\prime}} \int d \tau d \sigma \sqrt{-\dot{X}^{2} X^{\prime 2}+\left(\dot{X} \cdot X^{\prime}\right)^{2}}
\end{aligned}
$$

While one usually takes the limit $\rho_{\Lambda} \rightarrow \infty$, one may also keep $\rho_{\Lambda}$ finite and consider it as the energy the gauge theory is defined at. Note that the prescription given in (3.18) requires some sort of renormalization, usually given by the mass of the $\mathrm{W}$ bosons. This again is calculated from the action of a string stretching directly from the contour on the D7-brane to the bottom of the space as depicted in fig. 3(b). Note that this configuration is not physical, as it is not possible to define suitable boundary conditions at $\rho=a$. This will change in section 5.2, where we shall be considering the finite-temperature theory. Finite temperature is achieved by the presence of a black hole who's horizon gives suitable boundary conditions for the worldsheet in fig. 3(b) to be considered physical.

\subsubsection{Boundary conditions}

There is a crucial aspect of (3.18) that appears to be frequently overlooked. ${ }^{7}$ If we force the string to end on the contour $\mathcal{C}$, the resulting boundary conditions in at least some of the directions tangential to the brane are not von Neumann, but Dirichlet. One needs to ask for the object that restricts the string to lie on the contour.

As it is the easiest to understand this in terms of specific examples we shall delay explicit calculations to sections $3.5,5.2$, and 5.4.3. The technical aspects for all of these will be the same however, which is why we shall discuss them now.

Consider the Nambu-Goto action (3.19). It has a symmetry under translations

$$
X^{\mu} \rightarrow X^{\mu}+Y^{\mu} \quad Y^{\mu}=\text { const. },
$$

\footnotetext{
${ }^{7}$ See however [48].
} 
which we know from ordinary classical mechanics to be related to energymomentum conservation in space-time. Specialising to infinitesimal transformations, we can calculate the conserved current with the Noether prescription. As an intermediate result we obtain

$$
2 \pi \alpha^{\prime} j_{\mu}^{\alpha}=\frac{\partial \mathcal{L}}{\partial \partial_{\alpha} X^{\mu}}=g_{\mu \nu} \begin{cases}\frac{-\dot{X}^{\nu} X^{\prime 2}+X^{\prime \nu} \dot{X} \cdot X^{\prime}}{\sqrt{-\dot{X}^{2} X^{\prime 2}+\left(\dot{X} \cdot X^{\prime}\right)^{2}}} & \alpha=\tau \\ \frac{-\dot{X}^{2} X^{\prime \prime}+\dot{X}^{\nu} \dot{X} \cdot X^{\prime}}{\sqrt{-\dot{X}^{2} X^{\prime 2}+\left(\dot{X} \cdot X^{\prime}\right)^{2}}} & \alpha=\sigma\end{cases}
$$

$j_{\bar{\mu}}^{\tau}$ gives the energy $(\bar{\mu}=0)$ or $\bar{\mu}=\bar{m}$-momentum density on the string. $j_{\bar{\mu}}^{\sigma}$ on the other hand denotes the flux of energy or momentum along the string. Thus we can calculate the total energy and momentum to be

$$
\begin{aligned}
E & =\int d \sigma j_{\overline{0}}^{\tau} \\
P_{\bar{m}} & =\int d \sigma j_{\bar{m}}^{\tau} .
\end{aligned}
$$

The fluxes are related to an open string's boundary conditions. A string satisfying von Neumann boundary conditions does not allow for momentum to flow of the string, requiring

$$
\left.j_{\bar{\mu}}^{\sigma}\right|_{\text {boundary }}=0 .
$$

The solution of the issue of defining Dirichlet boundary conditions in directions tangent to a brane will be turning on $\mathcal{U}(1)$ gauge fields on the brane whose interaction with the string endpoints will exactly cancel the energy-momentum flow defined by these equations. The authors of [48] pointed out that as long as one keeps the position of the probe brane $\rho_{\Lambda}$ finite, it is more sensible to think of a constant force of the $\mathcal{U}(1)$ field on the string's endpoints rather than of a constant separation $L$ separating them.

\subsection{The quark-antiquark potential \& confinement}

Our first application of the concepts introduced in section 3.4 shall be the $q \bar{q}$ potential in the zero-temperature gauge theory. We follow [37]. Conceptually one studies this by placing two infinitively heavy and therefore static probequarks at a fixed separation $L$ into the gauge theory. For such a configuration, the action is independent of the time-like extension of the loop and therefore behaves as $S=E T$, with $E$ the energy of the system.

Now if the gauge theory is confining, the energy is proportional to $L$ from which it follows that

$$
E(L) \propto L \quad \Rightarrow \quad S \propto L T .
$$

$L T$ is the area surrounded by such a Wilson loop, so that for a confining theory we expect the action for the quark loop to satisfy an area law. ${ }^{8}$ In the following we shall study the $q \bar{q}$-potential of our gauge dual and whether it exhibits confinement.

\footnotetext{
${ }^{8}$ Technically (3.25) shows only that confinement leads to an area law. We are reversing the argument simply claiming that the converse is also true, i.e. that confinement occurs iff the action satisfies an area law. The relation between confinement and an area law for the Wilson loop was first discussed in [38].
} 


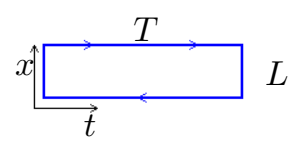

Figure 4: The rectangular Wilson loop used in section 3.5 as seen in the $(t, x)$ plane.

The profile In this section we will use the static Wilson loop shown in fig. 4 . Fixing $x \equiv x^{2}$, we may parametrize the loop and the corresponding worldsheet as

$$
x^{0}=\tau \quad x=\sigma \quad \rho=\rho(\sigma)
$$

where $\tau \in[0, T]$ and $\sigma \in\left[-\frac{L}{2}, \frac{L}{2}\right]$. Also we will need to impose the boundary conditions

$$
\rho(\sigma= \pm L / 2)=\rho_{\Lambda} .
$$

Note that the parametrization (3.26) does not define a complete Wilson loop but two Wilson lines separated by a distance $L$. Assuming $T \gg L$ however we may neglect the contribution from the pieces needed to close of the loop. Upon plugging (3.26) into the Nambu-Goto action (3.19) one notices immediately that the integration over $\tau$ is trivial giving an overall factor of $T$,

$$
S_{\mathrm{NG}}=\frac{T}{2 \pi \alpha^{\prime}} \int_{-\frac{L}{2}}^{\frac{L}{2}} d \sigma \underbrace{\sqrt{g_{t t}\left(g_{x x}+\rho^{\prime 2} g_{\rho \rho}\right)}}_{\mathcal{L}} .
$$

The idea is to treat this formally as a system from classical mechanics with Lagrangian $\mathcal{L}(\sigma)$. With $\sigma$ playing the role one would usually associate with time $t$ and identifying $\rho(\sigma)$ as the system's time coordinate, one calculates the canonical momentum $\pi$ and performs a Legendre transformation

$$
\begin{aligned}
\pi & =\frac{\partial \mathcal{L}}{\partial \rho^{\prime}} \\
\mathcal{H} & =\rho^{\prime} \pi-\mathcal{L}=\frac{-g_{x x} g_{t t}}{\sqrt{g_{t t}\left(g_{x x}+\rho^{\prime 2} g_{\rho \rho}\right)}} .
\end{aligned}
$$

From $\frac{\partial \mathcal{H}}{\partial \sigma}=0$ it follows with Hamilton's equations that $\frac{d \mathcal{H}}{d \sigma}=0$. Hence there is a conserved quantity

$$
\mathcal{H} \equiv \kappa \in \mathbb{R} .
$$

It might seem surprising that we emphasize that $\kappa$ is real. However we will encounter examples where this is not the case. As

$$
-g_{t t} g_{x x}=e^{\frac{4}{3} \Phi} \in\left[\left(12 N^{2}\right)^{-1}, \infty\right) \stackrel{N \rightarrow \infty}{\longrightarrow}[0, \infty),
$$

there exists $\rho_{c} \geq a$ s.t. $\kappa^{2}=-\left.g_{t t} g_{x x}\right|_{\rho=\rho_{c}}$. One sees immediately that $\left.\rho^{\prime}\right|_{\rho_{c}}=0$, which means that $\rho_{c}$ denotes the lowest point reached by the string. $\kappa=0$ holds if and only if the string reaches the bottom of the space. 
Solving (3.32) for $\rho^{\prime}$ yields a first order equation for the profile

$$
\rho^{\prime 2}=\frac{g_{x x}}{g_{\rho \rho}}\left(\frac{-g_{t t} g_{x x}-\kappa^{2}}{\kappa^{2}}\right) .
$$

Note that $g_{t t} \leq 0$. We assume the system to be symmetric about $\sigma=0$, which leads to the constraint $\rho^{\prime}(0)=0$. A look at the profile tells us that this is satisfied for

$$
\rho=\rho_{c} \geq a .
$$

See fig. 3(a). Note that $\rho^{\prime}$ is real as long as $\rho \geq \rho_{c}$.

Boundary conditions We briefly turn to the issue of the string's boundary conditions at the probe brane. Following the discussion in section 3.4.1 we are interested in the momentum flux at the endpoints of the string. Therefore we evaluate $j_{\mu}^{\sigma}$ as in (3.21) for the metric and profile in question and find

$$
j_{\mu}^{\sigma}=\frac{1}{2 \pi \alpha^{\prime}} \frac{\kappa}{g_{x x}}\left(\delta_{\mu}^{x} g_{x x}+\delta_{\mu}^{\rho} g_{\rho \rho} \rho^{\prime}\right) .
$$

The crucial observation is $j_{x}^{\sigma} \propto \kappa$. That is as long as the string does not reach the bottom of the space (i.e. $\rho_{c}=a$ ), there is momentum in the $x$-direction flowing through the string, violating von Neumann boundary conditions (3.24). We may easily fix this by turning on a $\mathcal{U}(1)$ gauge-field in the world-volume of the brane. Note that $\kappa \in \mathbb{R}$ tells us that one may choose the direction of momentum flow. This makes sense, as, the problem is symmetric and there is no reason a priori why the momentum should flow in a specified direction. We may interpret this as our freedom to choose which of the two heavy $\mathrm{W}$-bosons represents the quark and which represents the anti-quark. In other words while we set of with a mathematical model which was symmetric under a $q \leftrightarrow \bar{q}$ exchange, the appearance of the $\mathcal{U}(1)$ gauge field breaks this discrete symmetry.

$j_{\rho}^{\sigma}$ is also non-vanishing. Yet as $\rho$ denotes a direction transverse to the probe, this is in accordance with the Dirichlet boundary conditions in that direction.

Separation of the quarks $\rho_{c}$ is not a parameter but depends on the separation of the quarks. Regard

$$
L=2 \int_{0}^{\frac{L}{2}} d x=2 \int_{\rho_{c}}^{\rho_{\Lambda}} d \rho \rho^{-1} .
$$

One obtains a relation $L\left(\rho_{c}\right)$ which may be inverted to eliminate $\rho_{c}$. Albeit the integrand's singularity for $\rho \rightarrow \rho_{c}$, the integral is finite for fixed values of $\rho_{c}$ and $\rho_{\Lambda}$. For large $\rho$ however the integrand behaves roughly as

$$
\rho^{\prime-1} \stackrel{\rho \rightarrow \infty}{\approx} \frac{1}{\rho},
$$

s.t. one does not obtain a finite value for $L$ when taking $\rho_{\Lambda} \rightarrow \infty$. This is in contrast to asymptotically $A d S_{5}$ backgrounds, and might be related to the lack of a conformal boundary. 


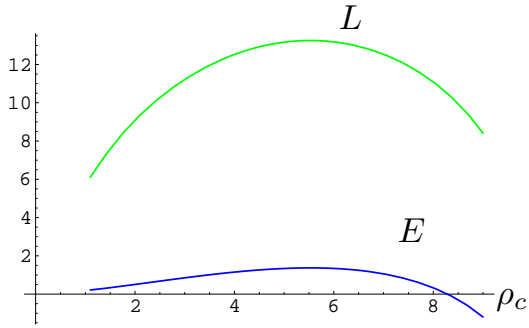

(a)

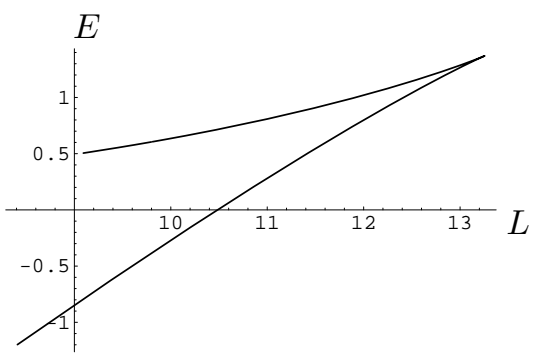

(b)

Figure 5: Separation $(L)$ and potential energy $(E)$ of the $q \bar{q}$ system for $a=1$, $\rho_{\Lambda}=10$, and $N=5$. $\rho_{c}$ denotes the lowest point in the bulk reached by the string. The $E(L)$ plot shows that for most values of $L$ there are 2 energy levels corresponding to a large and a small value of $\rho_{c}$. Minimizing its energy the system will choose the lower branch corresonding to larger values of $\rho_{c}$.

Renormalization As outlined in section 3.4 one renormalizes the action by evaluating the Nambu-Goto action for the worldsheet

$$
\tau=x^{0} \quad \sigma=\rho \quad x \in\left\{-\frac{L}{2}, \frac{L}{2}\right\} \quad \tau \in\{0, T\} .
$$

As with (3.26) this does not define a complete loop, but two separate lines. Again we may ignore this issue as long as we assume that $T \gg L$. Physically the overall procedure corresonds to subtracting the energy of two independent, static quarks. Proceeding as before, the counterterm is given by

$$
S_{\mathrm{R}}=\frac{T}{\pi \alpha^{\prime}} \int_{a}^{\rho_{\Lambda}} d \rho \sqrt{-g_{t t} g_{\rho \rho}} .
$$

One should emphasize again that, while being an admissible solution of the equations of motion, the solution used for renormalization here is not physical as there are no suitable boundary conditions to be defined at $\rho=a$. One should simply think of this as a method to calculate the mass of the W-bosons.

Evaluation Using $T E=S_{\mathrm{NG}}-S_{\mathrm{R}}$ and (3.19), (3.35), and (3.38) one obtains for the energy

$$
\begin{aligned}
E\left(\rho_{c}, \rho_{\Lambda}\right) & =\sqrt{-g_{t t}\left(\rho_{c}\right) g_{x x}\left(\rho_{c}\right)} L\left(\rho_{c}, \rho_{\Lambda}\right) \\
& +2 \int_{\rho_{c}}^{\rho_{\Lambda}} \sqrt{\frac{g_{\rho \rho}}{g_{x x}}}\left(\sqrt{-g_{t t} g_{x x}+g_{t t}\left(\rho_{c}\right) g_{x x}\left(\rho_{c}\right)}-\sqrt{-g_{t t} g_{x x}}\right) d \rho \\
& -2 \int_{a}^{\rho_{c}} \sqrt{-g_{t t} g_{x x}} d \rho .
\end{aligned}
$$

Numerical results are shown in figure 5 and show clearly that

$$
E(L) \propto L
$$

for $L \lesssim 13$. In order to properly exhibit confinement we would need to discuss the potential for $L>13$ in order to show that the proportionality holds for all values of $L$. 
As a matter of fact the behavior of $L$ around $L \approx 13$ stems from the fact that we did not take the $\rho_{\Lambda} \rightarrow \infty$ limit. That is, the separation between the branes is still finite and so is the mass of the probe quarks. Indeed, when running the same numerics for larger values of $\rho_{\Lambda}$, one ends up with similiar plots yet valid for larger values of $L$, which we take as a indication that the proportionality $E \propto L$ holds for any $L$. In order to properly establish confinement however, we shall use a different method. According to a theorem ${ }^{9}$ by Kinar, Schreiber, and Sonnenschein [37], a sufficient condition for confinement is given by the following: Consider the function

$$
f^{2}(\rho) \equiv-\left.g_{00} g_{x x}\right|_{\rho} .
$$

Then the dual gauge theory is confining if $f$ has a minimum at some $\rho_{\min }$ and $f\left(\rho_{\min }\right) \neq 0$. The metric (2.19) satisfies this and we conclude the discussion of the zero temperature theory by noting that the field theory is a confining.

\section{The supergravity theory at finite temperature}

Having completed our review of the zero-temperature theory, we shall discuss the finite-temperature case. Proceding in the same way as before, we begin with eleven-dimensional supergravity.

\subsection{The eleven-dimensional black hole}

Studying the quark gluon plasma means studying finite temperature physics. As for the gauge theory, finite-temperature field theory is - in the Matsubara formalism - defined on Euclidean space-time compactified to $S^{1} \times \mathbb{R}^{3}$. The previously time-like direction $x_{E}^{0}$ is now periodic with period $\beta=T^{-1}$. In the supergravity dual, we do also need to add an event-horizon to the background, turning the previously extremal p-brane solutions into non-extremal black branes [40]. One should picture this departure from extremality as adding energy to the background while keeping all charges constant. As the extremal solutions satisfy a BPS bound, adding temperature corresponds to using non-BPS branes. In order to do so, we modify the eleven-dimensional metric (2.1) to

$$
d s_{M}^{2}=-f(\rho) d t^{2}+d \mathbf{x}^{2}+\frac{d \rho^{2}}{f(\rho)\left(1-\frac{a^{3}}{\rho^{3}}\right)}+\ldots
$$

Note that we are using Minkowski-signature here, albeit the previous comments about the Matsubara formalism. The reason is that the procedure we use for finding the black brane solution does not depend on the signature and that we will be mostly using the Minkowski-space solution later on, because Euclidean time does not allow the study of dynamical quantities. However, in order to study genuinely themodynamical issues such as temperature, entropy, or specific heat, as we will do in section 4.2 , we need to compactifiy to periodic, Euclidean time.

Enforcing the equation of motion $R_{\mu \nu}=0$ on the above gives a system of differential equations for $f(\rho)$. While there will certainly be the trivial solution

\footnotetext{
${ }^{9}$ For a proof of the relevant theorem see [39].
} 
$f(\rho)=1$, we are looking for a nontrivial one exhibiting a horizon structure $f\left(\rho_{h}\right)=0$.

Calculating the Ricci tensor for the above ansatz one sees quickly that there is a non-trivial solution if and only if one takes $a \rightarrow 0$. While one might object that we are not allowed to take this limit as the zero temperature requires $a>0$ to resolve the conifold singularity, one should not forget that the singularity will be hidden by the black hole's horizon. The unique solution is

$$
f(\rho)=1-\frac{\rho_{h}^{5}}{\rho^{5}} .
$$

with

$$
\rho \in\left[\rho_{h}, \infty\right) .
$$

The new metric is given by

$$
d s_{M}^{2}=-f(\rho) d t^{2}+d \mathbf{x}^{2}+\frac{d \rho^{2}}{f(\rho)}+\frac{\rho^{2}}{9}\left(\tilde{w}^{a 2}+w^{a 2}-\tilde{w}^{a} w^{a}\right) .
$$

Most of our discussion will only require knowledge of the precise form of the $t, \mathbf{x}, \rho$ directions. When using Euclidean signature, we shall denote the metric by $\hat{g}_{\mu \nu}$.

\subsection{Thermodynamics}

We will now turn to a discussion of some of the thermodynamical properties of the solution (4.4).

Temperature Consider the $\left(t_{E}, \rho\right)$ plane in the finite-temperature formalism. It has topology $S^{1} \times \mathbb{R}_{>0}$ with $\rho \in\left[\rho_{h}, \infty\right)$ and $t_{E} \in[0, \beta]$. One proceeds by demanding that there be no conical singularity at the origin. Mathematically this may be expressed by considering the ratio of circumference and radius of a small circle around the origin and solving for

$$
2 \pi \stackrel{!}{=} \lim _{\rho \rightarrow \rho_{h}} \frac{\text { circumference }}{\text { radius }} .
$$

Using the standard expression for arclength, we obtain

$$
\begin{aligned}
& \text { circ. }=\int_{0}^{\beta} d t_{E} \sqrt{\hat{g}_{t t}} \approx \beta \rho \partial_{\rho} \sqrt{\hat{g}_{t t}(\rho)} \\
& \operatorname{rad} .=\int_{0}^{\rho} d \rho^{\prime} \sqrt{\hat{g}_{\rho \rho}} \approx \rho \sqrt{\hat{g}_{\rho \rho} .}
\end{aligned}
$$

Plugging these into (4.5) yields

$$
\begin{gathered}
2 \pi \stackrel{!}{=} \beta \lim _{\rho \rightarrow \rho_{h}} \frac{\beta \partial_{\rho} \sqrt{\hat{g}_{t t}}}{\sqrt{\hat{g}_{\rho \rho}}} \\
\Rightarrow T=\lim _{\rho \rightarrow \rho_{h}} \frac{\partial_{\rho} \hat{g}_{t t}}{4 \pi \sqrt{\hat{g}_{t t} \hat{g}_{\rho \rho}}}=\frac{5}{4 \pi \rho_{h}} .
\end{gathered}
$$

One should pay attention to the slightly unusual dependence of the temperature on the position on the horizon. For the $A d S_{5} \times S^{5}$ black hole for example, the relation is $T \propto \rho_{h}$. We will return to this issue in section 4.3. 
Evaluation of the partition function To study further thermodynamic properties of the solution (4.4), we need to evaluate the partition function $\mathcal{Z}=$ $e^{-\mathcal{S}_{E}}$. As the eleven dimensional theory is purely gravitational, this boils down to calculating the action

$$
\mathcal{S}=\frac{1}{16 \pi} \int_{\mathcal{M}} d^{d} x \sqrt{\hat{g}} R+\frac{1}{8 \pi} \int_{\partial \mathcal{M}} d^{d-1} x K \sqrt{\hat{h}}
$$

for Euclidean space-time. Where $\mathcal{M}$ is a volume of spacetime defined by $\rho<$ $\rho_{\Lambda}$. As in the absence of any further fields the equations of motion simplify to $R_{\mu \nu}=0$, the Einstein-Hilbert term vanishes leaving us with the GibbonsHawking term.

The metric induced on $\partial \mathcal{M}$ is denoted by $h . K$ is the extrinsic curvature defined by

$$
K_{a b} \equiv \partial_{a} x^{\mu} \partial_{b} x^{\nu} \nabla_{\mu} n_{\nu}
$$

The coordinates $x^{\mu}$ are that of the eleven-dimensional background, while the $x^{a}$ parametrize the boundary of the region of integration $\partial \mathcal{M}$. Due to our choice of volume $\mathcal{M}$ we may pick the $x^{a}$ such that

$$
\partial_{a} x^{\mu}= \begin{cases}\delta_{a}^{\mu} & \mu \neq \rho \\ 0 & \mu=\rho\end{cases}
$$

$n$ is a unit normal to $\partial \mathcal{M}$. We choose $n=\sqrt{g^{\rho \rho}} \partial_{\rho}$. Now (4.10) simplifies considerably.

$$
K_{a b}=\partial_{a} n_{b}-\Gamma_{\lambda a b} n^{\lambda}=-\Gamma_{\rho a b} \sqrt{g^{\rho \rho}}=\frac{1}{2} \sqrt{g^{\rho \rho}} \partial_{\rho} g_{a b}
$$

Similarly $h_{a b}=\partial_{a} x^{\mu} \partial_{b} x^{\nu} g_{\mu \nu}$ and thus

$$
\sqrt{h}=\frac{\rho^{6} \sqrt{f} \sin \theta \sin \tilde{\theta}}{648}
$$

Also

$$
K=h^{a b} K_{a b}=\frac{\sqrt{g^{\rho \rho}}}{2} g^{a b} \partial_{\rho} g_{a b}=\frac{\sqrt{f}}{2}\left(f^{-1} f^{\prime}+\frac{12}{\rho}\right) .
$$

Applying this to the action (4.9) one realizes that the integration is trivial as the radial variable is not integrated over. Then

$$
\begin{aligned}
\mathcal{S} & =\left.\overbrace{\left(\frac{1}{10368 \pi \sqrt{3}} \int d \mathbf{x} d \theta d \phi d d \tilde{\theta} d \tilde{\phi} d \tilde{\psi} \sin \theta \sin \tilde{\theta}\right)}^{\mathcal{A}} \int_{0}^{\beta} d x^{0} f \rho^{6}\left(f^{-1} f^{\prime}+\frac{12}{\rho}\right)\right|_{\rho=\rho_{\Lambda}} \\
& = \begin{cases}\mathcal{A} \beta\left(12 \rho_{\Lambda}^{5}-7 \rho_{h}^{5}\right) & T>0 \\
\mathcal{A} \beta 12 \rho_{\Lambda}^{5} & T=0\end{cases}
\end{aligned}
$$

Note that $\mathcal{A}=\frac{2 \pi^{3} \mathrm{Vol} \mathbb{R}^{3}}{81 \sqrt{3}}$. 
Renormalisation If we take the cutoff to infinity, $\rho_{\Lambda} \rightarrow \infty$, the result of (4.15) is divergent and does need to be renormalized. The easiest way to do so is by subtracting the action of some reference space-time. As we are only considering the Gibbons-Hawking term, the natural candidate is the zero-temperature solution as defined on the singular conifold, whose action may be obtained directly from (4.15) by setting $f \rightarrow 1$. We call this reference action $S_{T=0}$. We could have also calculated this reference action by starting from the non-singular zero-temperature metric (2.1), evaluating the on-shell action and taking the limit $a \rightarrow 0$ before $\rho_{\Lambda} \rightarrow \infty$.

Again we need to compactify the Euclidean $t_{E}$ direction on an $S^{1}$. Yet in opposite to the black hole solution (4.4) it is not obvious what the periodicity of the circle should be. Therefore consider a particle, whose energy is equal to the thermal energy $T$, in the finite-temperature solution propagating at a radius of $\rho_{\Lambda}$. To an observer at spatial infinity, its thermal energy will appear redshifted to

$$
E_{\infty}^{T}=\sqrt{-g^{t t}\left(\rho_{\Lambda}\right) p_{0} p_{0}}=\frac{T}{\sqrt{\hat{g}_{t t}\left(\rho_{\Lambda}\right)}}=\frac{T}{\sqrt{f\left(\rho_{\Lambda}\right)}} .
$$

In the zero temperature solution on the other hand, $\hat{g}_{t t}=1$, and there is no redshift. Comparing energies in the two solutions by means of hypothetical observers at $\rho=\infty$, the energies correspond as

$$
E_{\rho_{\Lambda}}^{T=0}=\frac{E_{\rho_{\Lambda}}^{T}}{\sqrt{f\left(\rho_{\Lambda}\right)}},
$$

which leads us to

$$
\beta_{T=0}=\beta_{T} \sqrt{1-\frac{\rho_{h}^{5}}{\rho_{\Lambda}^{5}}} .
$$

We shall use this result to evaluate and compare (4.15) for the zero- and finite-temperature backgrounds with $t_{E}$ periodic and periodicity $\beta_{T=0}, \beta_{T}$, yielding

$$
\begin{aligned}
& \mathcal{S}_{T=0}=12 \mathcal{A} \beta \rho_{\Lambda}^{5} \sqrt{1-\frac{\rho_{h}^{5}}{\rho_{\Lambda}^{5}}} \\
& \mathcal{S}_{T>0}=-7 \mathcal{A} \beta \rho_{h}^{5}+12 \beta \mathcal{A} \rho_{\Lambda}^{5} .
\end{aligned}
$$

Taking the cutoff $\rho_{\Lambda}$ to infinity, evaluating $\mathcal{A}$ explicitly, and dividing by the volume of $\mathbb{R}^{3}$, the final, renormalized result for the action density is

$$
\mathcal{S}_{E}=\lim _{\rho_{\Lambda} \rightarrow \infty} \mathcal{S}_{T>0}-\mathcal{S}_{T=0}=-\frac{8 \pi^{4} \rho_{h}^{6}}{405 \sqrt{3}} .
$$

The fact that this seems to be negative should not disturb us. In the contrary, as it implies $s_{T=0}>s_{T>0}$, the finite temperature solution will be the leading order contribution in a saddle point approximation to the path integral. If this was not the case, we were not allowed to study finite temperature effects using the solution (4.4). Naturally, when computing further quantities, we will use the absolute value of (4.21).

One should wonder about the $N$ dependence of (4.21). After all our aim is to study the physics of the QGP, which is in a deconfined phase of QCD. 
So the entropy should reflect the $N^{2}$ color-degrees of freedom. On the other hand, (4.21) cannot contain any factor $N$, as the UV completion does not know about the number of colors. One may try to resolve this issue by substituting the 't Hooft coupling $\lambda$ for $\rho_{h}$. We will compute $\lambda$ in section 5.1, yet for our discussion here it is sufficient to know that when expressed in terms of $N, \lambda$, and energy-scale $\rho, \rho_{h}$ has a

$$
\rho_{h}^{5} \sim \frac{N^{5}}{\lambda}
$$

dependence, leading to a $N^{6} \lambda^{-6 / 5}$ dependence for the entropy. While this is not fully satisfactory - after all, one would expect $N^{2}$, it shows the correct qualitative behavior.

Mass, Entropy, Specific heat Using the renormalized Euclidean action (4.21) and some standard relations of thermodynamics one can calculate a variety of properties of the background. Mass, entropy-density and specific heat are given by

$$
\begin{aligned}
\mathcal{Z} & =e^{-\mathcal{S}_{E}} \\
M & =\langle E\rangle=\frac{\partial \mathcal{S}_{E}}{\partial \beta}=\frac{5}{4 \pi} \frac{\partial \mathcal{S}_{E}}{\partial \rho_{h}} \\
S & =\beta\langle E\rangle-\mathcal{S}_{E} \\
C & =T \frac{\partial S}{\partial T} .
\end{aligned}
$$

Therefore

$$
\begin{aligned}
M & =\frac{4 \pi^{3} \rho_{h}^{5}}{27 \sqrt{3}} \\
S & =\frac{8 \pi^{4} \rho_{h}^{6}}{81 \sqrt{3}} \\
C & =-\frac{16 \pi^{4} \rho_{h}^{6}}{27 \sqrt{3}} .
\end{aligned}
$$

Equations (4.27) show a rather surprising thermodynamic behavior - especially as we are trying to identify it with that of a four-dimensional gauge theory. First of all, the specific heat $C$ is negative, probably denoting an instability of the solution. More importantly, the entropy behaves as $S \propto T^{-6}$, which is rather puzzling. As a first check of the above results, one can compare (4.27) to the Bekenstein-Hawking entropy, which in our conventions takes the form $S_{\mathrm{BH}}=\frac{A}{4}$, with $A$ being the area of the black hole horizon. A direct calculation gives $S_{\mathrm{BH}}=\frac{8 \pi^{4}}{81 \sqrt{3}} \rho_{h}^{6}$, which agrees with the previous result. One should also note that the first law of thermodynamics, $d M=T d S$, is satisfied by the solution, as can be verified explicitly.

So while the thermodynamical properties of the system appear sensible from the point of view of eleven-dimensional supergravity, it is difficult to interpret them as those of a four-dimensional gauge theory. We will try to find a partial explanation for this behavior in the next section. 


\subsection{Comparison with the Schwarzschild solution}

In comparison with the AdS-black hole [40] properties of the finite-temperature $G_{2}$ holonomy solution (4.4) might seem a bit surprising. However, there is a very well understood solution of the four-dimensional Einstein equations with similiar characteristics, the Schwarzschild black hole. So let us recall the properties of its generalization, the four-dimensional Reissner-Nordstrom solution.

$$
\begin{aligned}
& d s^{2}=-\left(1-\frac{2 M}{r}+\frac{Q^{2}}{r^{2}}\right) d t^{2}+\left(1-\frac{2 M}{r}+\frac{Q^{2}}{r^{2}}\right)^{-1} d r^{2}+r^{2} d \Omega_{2}^{2} \\
& r_{ \pm}=M \pm \sqrt{M^{2}-Q^{2}} \quad T=\frac{1}{4 \pi}\left(\frac{2 M}{r_{+}^{2}}-\frac{2 Q^{2}}{r_{+}^{3}}\right) \quad F=\frac{Q}{r^{2}} d t \wedge d r
\end{aligned}
$$

$M$ is the mass, $Q$ the charge, $T$ the temperature, and $r_{ \pm}$are the inner and outer horizons. The Schwarzschild solution is obtained in the $Q \rightarrow 0$ limit. As one may see from the equations, there is a BPS constraint on the mass $M \geq Q$.

As long as we keep $Q>0$, the temperature vanishes in the extremal limit $M \rightarrow Q$. This changes in the Schwarzschild case $Q=0$. Here the temperature is singular when taking the mass to zero. Mathematically this is expressed by the absence of the $+\frac{Q^{2}}{r^{2}}$ term in the Schwarzschild metric. As there is no such term in the eleven-dimensional metric (4.4) and as both the Schwarzschild and the Reissner-Nordstrom solution have negative specific heat ${ }^{10}$, one may speculate that the singular behavior of the temperature of the gravity dual in question may be related to the dual being of Schwarzschild- rather than Reissner-Nordstrom type.

We may pursue the comparison with the Schwarzschild solution even further. Our zero-temperature background has the topology $\mathbb{R}^{1,3} \times \mathbb{R} \times \mathcal{M}$, with $\mathcal{M}$ being the $G_{2}$-holonomy manifold. If we were simply to replace $\mathcal{M}$ by an $S^{6}$, we were dealing with ordinary Minkowski space in eleven dimensions. Now searching for a black hole of with the Ansatz

$$
d s^{2}=-f(\rho) d t^{2}+d \mathbf{x}^{2}+\frac{d \rho}{f(\rho)}+\rho^{2} d \Omega_{6}^{2}
$$

we find the identical solution to the equations of motion, $R_{\mu \nu}=0$, given by (4.2). Performing the same calculations on this eleven-dimensional Schwarzschild black hole that we did before, we see, that the Bekenstein-Hawking entropy behave as $S_{\mathrm{BH}} \propto \rho_{h}^{6}$, whereas the temperature will satisfy $T=\frac{5}{4 \pi \rho_{h}}$, showing thermodynamic behavior identical to that of our solution (4.4). Thus it appears as if the rather undesirable behavior of the entropy $S \propto T^{-6}$ might be related to the fact that the string dual may be traced back to pure gravity in eleven dimensions. In analogy with the four-dimensional case one might expect the thermodynamics of our solution to improve once the black hole is charged under some gauge field. Generalizing the ansatz (4.1) to include the three-form potential of eleven-dimensional supergravity however will make the task of finding a solution considerably more difficult.

\footnotetext{
${ }^{10}$ For Schwarzschild one sees this by realizing that any increase in $M$ leads to a decrease in $T$. So whenever we increase the energy, keeping the charge constant, the temperature decreases. For Reissner-Nordstrom the situation is slightly more complicated. While $T$ vanishes with $M$ for sufficiently small $M$ the behavior reduces to that of Schwarzschild in the large $M$ limit. It follows that Reissner-Nordstrom black holes of small masses have positive specific heat, while those of large mass have negative specific heat.
} 


\subsection{Dimensional Reduction}

In the same way that we went from M-theory to type IIA at zero temperature in section 2.2 , one may perform dimensional reduction for the finite-temperature background.

$$
\begin{aligned}
e^{\frac{4 \Phi}{3}} & =\frac{\rho^{2}}{9 N^{2} \rho_{h}^{2}} \\
A_{(1)} & =N \rho_{h}\left(n . \hat{w}-\frac{1}{2} n . w\right) \\
d s_{I I A}^{2} & =e^{\frac{2}{3} \Phi}\left[-f(\rho) d t^{2}+d \mathbf{x}^{2}+\frac{d \rho^{2}}{f(\rho)}+\frac{\rho^{2}}{9}\left(w^{2}+\hat{w}^{2}-w \cdot \hat{w}\right)-e^{\frac{4}{3} \Phi} A_{(1)} A_{(1)}\right]
\end{aligned}
$$

The Ricci scalar in the string frame is

$$
R=\frac{9 N \rho_{h}}{\rho^{8}}\left(-13 \rho^{5}+3 \rho_{h}^{5}\right) .
$$

\section{The field theory at finite temperature}

\subsection{Properties of the Dual Field Theory}

Turning on a temperature does naturally break the supersymmetry, so that we are dealing with the same modes as in the zero-temperature case, except that there is no supersymmetry. Now however the mass of the Kaluza-Klein modes is given by the size of the wrapped $S^{3}$ in the far IR, that is by the location of the horizon. We may use (4.8) to relate it to the temperature as

$$
\Lambda_{K K}=\frac{\alpha^{\prime}}{2 \pi^{2} \rho_{h}^{3}}=\frac{1}{2}\left(\frac{4}{5}\right)^{3} \alpha^{\prime} T^{3}
$$

In all other aspects the discussion of the theory's field content is identical to that performed in section 3.2.

The same holds true for the derivation of the Yang-Mills coupling constant from the DBI action (3.1). The induced metric is

$$
\begin{aligned}
d s_{6}^{2}=e^{\frac{2}{3} \Phi} & {\left[-f d t^{2}+d \mathbf{x}^{2}+\frac{\rho^{2}}{9} d \tilde{\theta}^{2}+\frac{\rho^{2}}{12} d \tilde{\phi}^{2}+\frac{\rho^{2}}{9}\left(1-\frac{1}{4} \cos ^{2} \tilde{\theta}\right) d \tilde{\psi}^{2}\right.} \\
& \left.+2 \frac{\rho^{2}}{12} \cos \tilde{\theta} d \tilde{\phi} d \tilde{\psi}\right]
\end{aligned}
$$

leading to

$$
g_{Y M}=\frac{3^{13 / 4} N \pi \alpha^{\prime 3 / 4} \rho_{h}}{\rho^{5 / 4}\left(\rho^{5}-\rho_{h}^{5}\right)^{1 / 4}}
$$

Having already calculated the dilaton (4.31) and the Ricci scalar (4.34), we are again able to discuss the decoupling limit. To get a qualitative understanding 


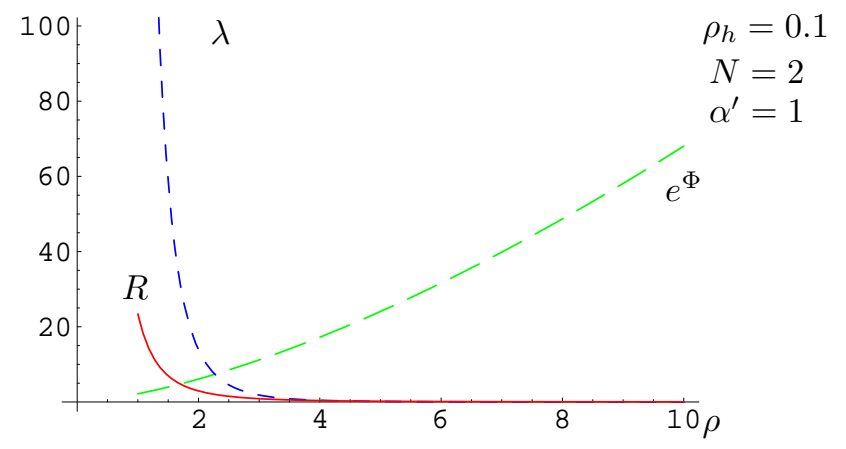

Figure 6: As previously done for the zero temperature gauge theory in figure 2, we discuss the curvature and couplings of the finite temperature solution. Again there is clearly a regime in the IR where non-perturbative gauge-dynamics are captured by type IIA string theory. As in the zero-temperature case though, $g_{Y M}$ is singular at $\rho=\rho_{h}$.

we have plotted the relevant quantities in fig. 6 .

$$
\begin{aligned}
-\alpha^{\prime} R & \leq \frac{90 N \alpha^{\prime}}{\rho_{h}^{2}} \\
\lambda & =\frac{324 \sqrt{3} N^{3} \pi^{2} \alpha^{3 / 2} \rho_{h}^{2}}{\rho^{5 / 2} \sqrt{\rho^{5}-\rho_{h}^{5}}} \\
e^{\Phi} & =\left(\frac{\rho}{3 N \rho_{h}}\right)^{\frac{3}{2}}
\end{aligned}
$$

Again the supergravity description is valid in the large $N$, small $\alpha^{\prime}$ limit while it is not possible to ignore the KK-modes ( $\rho_{h}$ small $)$ at the same time.

\subsection{Quark-Antiquark Potential}

We perform a numerical analysis of the quark-antiquark potential. The results presented here were derived in exactly the same way as in section 3.5 with the finite temperature metric (4.33) replacing the zero temperature background (2.19).

The results are depicted in fig. 5. At first sight it appears as if there are again two solutions with the minimum energy one showing a direct proportionality $E \propto L$ and thus confinement. If this were the complete story the physical system dual to our finite-temperature background were certainly not a deconfined QGP.

Now recall from our discussion of the Wilson loop's renormalization in sections 3.4 and 3.5 that for the zero temperature solution the configuration of two strings stretching from the probe brane to the bottom of the space $(\rho=a)$ was not physical as it is not possible to define suitable boundary conditions for the worldsheet. In other words, there is nothing at the bottom of the space for the open strings to end on. This is different for the finite temperature case though, where it is possible for a string to end (or fall through) a black hole's horizon, as long as suitable boundary conditions are satisfied; i.e. there may be no excitations leaving the black hole. Therefore renormalization in the finite temperature 


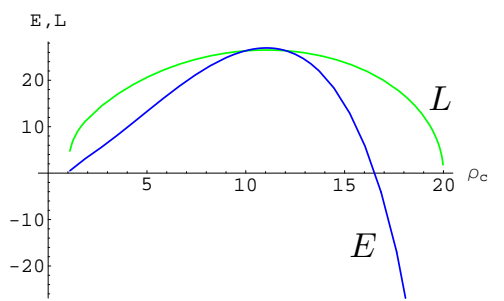

(a)

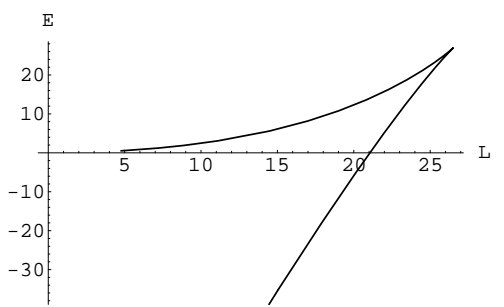

(b)

Figure 7: The quark-antiquark potential at finite temperature. Compare the zero temperature case shown in figure 5 .

theory is not interpreted as merely subtracting the mass of the two W-bosons. Instead one actually considers two competing, physical solutions. That of two quarks connected by a string and that of two independent quarks. The system chooses the minimum energy configuration and therefore we may interpret the point in fig. 7 (b) at $L \approx 21$ where $E(L)=0$ as the transition between the two solutions. For $L>21$ we have two quarks propagating independently, ${ }^{11}$ while for $L<21$ the two quarks interact via a string. Therefore we claim that the finite temperature theory is not confining, as expected for the QGP.

As to the issue of the world-sheet's boundary conditions, the discussion is identical to that of the zero temperature case in section 3.5. The $x$-momentum flux along the string is proportional to a constant of integration $\kappa$ with $\kappa=0$ if and only if the string stretches all the way to the horizon. Again one fixes the failure of the boundary conditions to be properly von Neumann by turning on a $\mathcal{U}(1)$ gauge field in the probe brane.

\subsection{Shear Viscosity}

One of the first properties of the $\mathcal{N}=4$ QGP calculated from the dual $A d S_{5} \times S^{5}$ geometry was the plasma's shear viscosity $\eta \cdot{ }^{12}$ The original ansatz of [8] uses the Kubo relations which stem from the formalism of finite-temperature field theory. These relate the shear viscosity to the energy-momentum tensor as

$$
\eta=\lim _{\omega \rightarrow 0} \frac{1}{2 \omega} \int d t d \mathbf{x} e^{\imath \omega t}\left\langle\left[T_{x y}(t, \mathbf{x}), T_{x y}(0,0)\right]\right\rangle .
$$

While one may simply use the gauge/gravity correspondence to directly calculate the above correlator, the authors of [41] were able to identify hydrodynamic behavior in the gravity dual by studying metric perturbations in the background. Thus they obtained an explicit expression for the shear viscosity in terms of the entropy density. Defining $g=\operatorname{det} g_{\mu \nu}$,

$$
\frac{\eta}{s}=\left.T \frac{\sqrt{-g}}{\sqrt{-g_{00} g_{\rho \rho}}}\right|_{\rho_{h}} \int_{\rho_{h}}^{\infty} d \rho \frac{-g_{00} g_{\rho \rho}}{g_{x x} \sqrt{-g}} .
$$

\footnotetext{
${ }^{11}$ The quarks are not fully independent. The two worldsheets interact via graviton exchange in the bulk spacetime.

${ }^{12}$ Brief reviews of relativistic hydrodynamics and their relevance to relativistic heavy ion collisions may be found in $[13,14]$.
} 


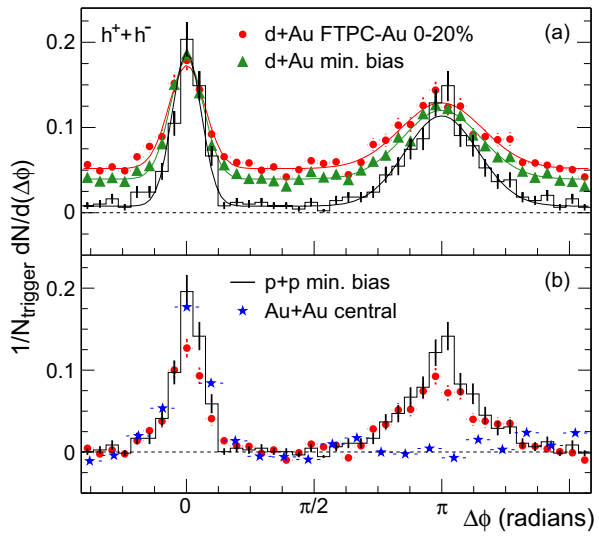

Figure 8: Experimental evidence for jet quenching in heavy ion collisions (Source: [44]).

Evaluating the above for the type IIA or 11-dimensional background (4.33), (4.4) yields

$$
\frac{\eta}{s}=\frac{1}{4 \pi}
$$

The above result confirms a general theorem [42], [43] according to which the ratio $\eta / s=1 / 4 \pi$ is of the same value for a fairly large class of gravity duals.

\subsection{Energy Loss of a Heavy Quark}

Our final object of study shall be the radiative energy loss of a heavy quark traversing the plasma. Prior to exhibiting how this may be modeled in terms of the AdS/CFT correspondence and the $G_{2}$ holonomy manifold we shall take a brief excursion into experimental data obtained at the relativistic heavy ion collider in order to see why radiative energy loss is a problem of interest.

\subsubsection{Experimental Background}

The relativistic heavy ion collider performs central $\mathrm{Au}+\mathrm{Au}$ collisions at about $200 \mathrm{GeV}$. After the collision the system quickly reaches a local thermal equilibrium at a temperature of about $170 \mathrm{MeV}$ and is assumed to be a quark-gluon plasma. ${ }^{13}$ Naturally the plasma is not the only result of the collision. Instead there is also a number of partons whith energies of up to $\mathcal{O}(1 \mathrm{GeV})$. One might expect that these should be created in two- or three-jet events. Specializing to back-to-back scattering, figure 8(b) shows the yield of such partons in terms of their angular distribution in the reaction plane. The concept is to wait for a trigger particle with transverse momentum $4<p_{T \text {,Trig. }}<6 \mathrm{GeV} / c$ and then search for further particles with $2 \mathrm{GeV} / c<p_{T \text {,Trig. }}$ With the trigger particle at $\Delta \Phi=0$ one sees clearly a suppression of such back-to-back events in the $\mathrm{Au}+\mathrm{Au}$ heavy ion collisions in comparision to ordinary $\mathrm{p}+\mathrm{p}$ scattering. The reason for this suppression lies in the fact that, as sketched in figure 9, one of the partons needs to traverse the plasma. In doing so it interacts with the plasma leading

\footnotetext{
${ }^{13}$ For a review of relativistic heavy ion collisions see [13].
} 


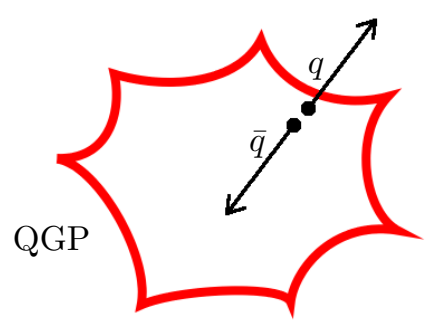

Figure 9: Jet Quenching in Relativistic Heavy Ion Collisions is due to radiative energy loss of a parton - here the antiquark $\bar{q}$ - traversing the plasma.

to an overall energy loss. The answer to our initial question should be clear from this: As this phenomenon is specific to heavy ion collisions, it may be directly attributed to the presence of the plasma and is therefore an experimental indicator to the QGP being created in the course of the experiment.

When applying the AdS/CFT-correspondence to describe parton energy loss, there are two fundamentally different approaches. One, referred to in the literature as the jet quenching calculation [10],[17] models the problem in terms of ordinary particle physics and uses the correspondence exclusively for purposes of computation. The concept of the drag force on the other hand is intrinsically stringy as the quark is depicted as a string hanging from a probe brane into the bulk geometry $[11,45]$. There is a further difference between the two approaches. While the former relies on the energy of the quark being highly relativistic, the latter is not only free of this assumption but is moreover frequently used to make statements about the non-relativistic limit.

\subsubsection{Jet Quenching}

In the jet quenching picture, the energy loss of the high energy quark is captured by the jet quenching parameter $\hat{q}$ which again is defined in terms of the expectation value of a Wilson loop:

$$
\langle W(\mathcal{C})\rangle=e^{-\frac{1}{4} \hat{q} L^{-} L^{2}}
$$

Here $\mathcal{C}$ is a light-like Wilson loop in the $x^{2}, x^{-}=\frac{x^{0}-x^{3}}{\sqrt{2}}$ plane. The extension along the light-cone is $L^{-}$while that along $x^{2}$ is $L$. One assumes $L^{-} \gg L$. One should note that albeit the loop being defined in Minkowski space, the exponential on the right hand side of (5.10) is a real quantity. This is in contrast to (3.18), which is defined in Euclidean space.

The derivation of (5.10) is purely based on particle theory and rather nontrivial. We shall only briefly describe how $\hat{q}$ captures the phenomenon of radiative energy loss and why one may use a Wilson loop to calculate it. The interested reader is referred to the literature [46], [50] for details on how (5.10) arises.

To answer the first of these questions, note that parton energy loss is directly proportional to the jet quenching factor,

$$
\Delta E \propto \hat{q} L^{-2} .
$$


As to the question of why this may be calculated using a Wilson loop, consider the following: Due to the quarks high energy, we may think of it as actually moving along the light-cone. Interaction with the gluons of the plasma leads to color rotations. One may think of in- and out-states related by a Wilson line along the light-cone

$$
\left|\Psi_{\text {out }}\right\rangle=\operatorname{Tr} \mathcal{P} e^{\imath \int_{0}^{L^{-}} d x_{-} A^{-}}\left|\Psi_{\text {in }}\right\rangle .
$$

Expectation values involve the hermitian conjugate of this, leading to a Wilson line in the opposite direction. As $L^{-} \gg L$, one may join the two lines giving us the loop $\mathcal{C}$.

Taking a closer look at (5.10), a crucial observation is that we are dealing with the exponenential of a real quantity albeit using Minkowskian signature. This is directly related to the occurence of the light-like Wilson loop. Although it is technically possible to obtain a result for the jet-quenching factor using such a loop, as was done in [17], we will see that one needs to consider such a light-like loop as the limiting case of space- or time-like ones extending either down- or upwards from the flavor brane they are attached to. Note that while the original paper [46] considered only a space-like string stretching from the flavor brane towards the horizon and approaching the light-like limit from below, $v<1$, it was argued in $[47,48,49]$ that all four cases need to be investigated. As the technicalities follow analogous steps in all four cases, we will only exhibit a detailed calculation for the space-like down string followed by some remarks about the three remaining configurations.

The space-like down-string We consider the quark-qntiquark pair as moving with constant speed $v=\tanh \eta$. Eventually we will take the limit $v \rightarrow 1$. At first we will assume the string to stretch from the flavor brane at $\rho_{\Lambda}=\Lambda \rho_{0}$ towards the horizon at $\rho_{h}$. We are interested in the limit $\Lambda \rightarrow \infty$, the case of infitively heavy quarks. Moving to a coordinate frame in which the pair lies at rest leads to a new metric given by

$$
\begin{aligned}
g_{00}^{\prime} & =\frac{\rho}{3 N \rho_{h}}\left[-f \cosh ^{2} \eta+\sinh ^{2} \eta\right] \\
& =-\frac{\rho}{3 N \rho_{h}}\left[1-\left(\frac{\rho_{h}}{\rho}\right)^{5} \cosh ^{2} \eta\right] \\
g_{x^{3} x^{3}}^{\prime} & =\frac{\rho}{3 N \rho_{h}}\left[\cosh ^{2} \eta-f \sinh ^{2} \eta\right] \\
g_{0 x^{3}}^{\prime} & =\frac{\rho}{3 N \rho_{h}}[-f \cosh \eta \sinh \eta+\cosh \eta \sinh \eta]
\end{aligned}
$$

with the other components as before in (4.33). As $x^{3}$ will not appear in our calculations, we shall ignore the primes from now on and define $x \equiv x^{2}$. In these coordinates the profile is that of a static quark-antiquark pair and therefore the same as in (3.26) in section 3.5. Note that if the elongation along $x^{0}=t$ in this reference frame is $\mathcal{T}$, then it is $L^{-}=\mathcal{T} \cosh \eta$ in the laboratory frame.

The Nambu-Goto action is

$$
S_{\mathrm{NG}}=\frac{\mathcal{T}}{\pi \alpha^{\prime}} \int_{0}^{\frac{L}{2}} d \sigma \sqrt{-g_{00}\left(g_{x x}+\rho^{\prime 2} g_{\rho \rho}\right)} .
$$


Ignoring the overall normalisation,

$$
\begin{aligned}
\mathcal{L} & =\sqrt{-g_{00}\left(g_{x x}+\rho^{\prime 2} g_{\rho \rho}\right)} \\
& =\sqrt{\frac{\rho}{3 N \rho_{0}}\left[1-\left(\frac{\rho_{0}}{\rho}\right)^{5} \cosh ^{2} \eta\right]\left(g_{x x}+\rho^{\prime 2} g_{\rho \rho}\right)} .
\end{aligned}
$$

While the second term is positive definite, the first term however might change sign, depending on the values of $\eta$ and $\Lambda$, with the $\Lambda$ dependence arising as $\rho \in\left\{\rho_{0}, \Lambda \rho_{0}\right\}$. We see that as long as

$$
\cosh ^{2} \eta>\Lambda^{5},
$$

the Lagrangian $\mathcal{L}$ is imaginary. This is what guarantees the exponent in (5.10) to be real, as required. Therefore the limits $\eta \rightarrow \infty$ and $\Lambda \rightarrow \infty$ do not commute.

The Hamiltonian is

$$
\mathcal{H}=\frac{g_{00} g_{x x}}{\mathcal{L}} \equiv \kappa \quad \kappa \in \imath \mathbb{R}
$$

In the problem in question $\kappa$ is purely imaginary, as the Lagrangian is imaginary. The profile is given by

$$
\begin{aligned}
\rho^{\prime 2} & =\frac{g_{x x}}{g_{\rho \rho}}\left(\frac{-g_{00} g_{x x}-\kappa^{2}}{\kappa^{2}}\right) \\
& =\frac{f}{\kappa^{2}}\left\{\frac{\rho^{2}}{9 N^{2} \rho_{h}^{2}}\left[1-\left(\frac{\rho_{h}}{\rho}\right)^{5} \cosh ^{2} \eta\right]-\kappa^{2}\right\}
\end{aligned}
$$

with $\kappa^{2} \leq 0$. For this to be real and positive, one needs to impose constraints on $\kappa$.

$$
\cosh ^{2} \eta-\Lambda^{5}-9 N^{2} \Lambda^{3}|\kappa|^{2} \geq 0
$$

So from now on we shall assume $|\kappa| \ll 1$.

Evaluating the length and the action We choose new coordinates,

$$
\rho=\rho_{h} y \quad L=\rho_{h} l .
$$

Then

$$
\begin{aligned}
l & =\frac{2}{\rho_{h}} \int_{0}^{\frac{L}{2}} d x=\frac{2}{\rho_{h}} \int_{\rho_{h}}^{\Lambda \rho_{h}} d \rho \rho^{\prime-1} \\
& =2|\kappa| \int_{1}^{\Lambda} d y\left(\frac{y^{8}}{y^{5}-1}\right)^{\frac{1}{2}}\left(\frac{9 N^{2}}{\cosh ^{2} \eta-y^{5}-|\kappa|^{2} 9 N^{2} y^{3}}\right)^{\frac{1}{2}}
\end{aligned}
$$


We are interested in the small $l$ behavior, which is equivalent to assuming $\kappa$ to be small. Expanding the integrand gives

$$
\begin{aligned}
& =6 N|\kappa| \int_{1}^{\Lambda} d y \frac{y^{4}}{\sqrt{y^{5}-1}}\left(\frac{1}{\sqrt{\cosh ^{2} \eta-n y^{5}}}+\frac{|\kappa|^{2} 9 N^{2} y^{3}}{2\left(\cosh ^{2} \eta-y^{5}\right)^{3 / 2}}+\mathcal{O}\left(|\kappa|^{4}\right)\right) \\
& =6 N|\kappa| \underbrace{\int_{1}^{\Lambda} d y \frac{y^{4}}{\sqrt{y^{5}-1}} \frac{1}{\sqrt{\cosh ^{2} \eta-y^{5}}}}_{A}+\mathcal{O}\left(|\kappa|^{3}\right) \\
& =\frac{6 N}{\cosh \eta}|\kappa| \underbrace{\int_{1}^{\Lambda} d y \frac{y^{4}}{\sqrt{y^{5}-1}}}_{B}+\mathcal{O}\left(|\kappa|^{3}, \frac{1}{\cosh \eta}\right)
\end{aligned}
$$

In the last equation we assumed that $\frac{\Lambda^{5}}{\cosh ^{2} \eta}$ is sufficiently small in order to develop the expression in $\cosh ^{-1} \eta$. In the $\Lambda \rightarrow \infty$ limit, the integral $B$ is certainly divergent, which might raise the question wheter $l$ may truly be considered to be small. Closer examination however shows that for large $y$,

$$
B \sim \Lambda^{\frac{5}{2}} \cosh ^{-1} \eta
$$

As $\cosh ^{2} \eta \geq \Lambda^{5}$, our assumption about $l$ is justified.

Similarly to the lenght we may treat the action,

$$
\begin{aligned}
S_{\mathrm{NG}} & =\frac{\imath \mathcal{T}}{\pi \alpha^{\prime}} \int_{\rho_{h}}^{\Lambda \rho_{h}} d \rho \rho^{\prime-1} \sqrt{\frac{g_{00}^{2} g_{x x}^{2}}{|\kappa|^{2}}} \\
& =\frac{\imath \mathcal{T} \rho_{0}}{3 \pi \alpha^{\prime} N} \int_{1}^{\Lambda} d y \sqrt{\frac{y^{2}\left(\cosh ^{2} \eta-y^{5}\right)^{2}}{y^{5}-1}} \frac{1}{\sqrt{\cosh ^{2} \eta-y^{5}-|\kappa|^{2} y^{3} 9 N^{2}}} \\
& =\frac{\imath \mathcal{T} \rho_{0}}{3 \pi \alpha^{\prime} N} \int_{1}^{\Lambda} d y \sqrt{\frac{y^{2}\left(\cosh ^{2} \eta-y^{5}\right)}{y^{5}-1}} \\
& +\frac{3 \imath \mathcal{T} N \rho_{0}|\kappa|^{2}}{2 \pi \alpha^{\prime}} \underbrace{\int_{1}^{\Lambda} d y \frac{y^{4}}{\sqrt{y^{5}-1}} \frac{1}{\sqrt{\cosh ^{2} \eta-y^{5}}}}_{A}+\mathcal{O}\left(|\kappa|^{4}\right) \\
& \equiv S^{(0)}+|\kappa|^{2} S^{(1)}+\mathcal{O}\left(|\kappa|^{4}\right)
\end{aligned}
$$

If one again only looks into the leading order behavior for $\cosh ^{-1} \eta$, the $\mathcal{O}\left(|\kappa|^{2}\right)$ term is

$$
S^{(1)}=\frac{3 \imath \mathcal{T} N \rho_{0}|\kappa|^{2}}{2 \pi \alpha^{\prime} \cosh \eta} \underbrace{\int_{1}^{\Lambda} d y \frac{y^{4}}{\sqrt{y^{5}-1}}}_{B}=\imath \frac{T L^{2} L^{-}}{30 \alpha^{\prime} B}
$$

Note the reappearance of the integrals $A, B$. Renormalizing the above action as described in section 3.4 yields a counterterm that exactly cancels $S^{(0)}$. So to first order in $|\kappa|^{2}$ we may work with $S^{(1)}$. 
The remaining configurations \& jet quenching From equation (5.21) it follows that one may also consider a world-sheet ending on the flavor brane yet stretching away from the horizon s.t. $\rho \geq \Lambda \rho_{h}$. Using the same approximations as for the down-string of the previous paragraph, one arrives at an expression identical to (5.28) except for the integration bounds. Once more, taking $\eta \rightarrow \infty$ before $\Lambda \rightarrow \infty$, the relevant integral $B$ diverges.

For the string with $v>1$ one boosts to a faster than light frame. Technically this amounts to substituting $\cosh \eta \mapsto \frac{1}{2 \sinh \zeta}$ and $\sinh \eta \mapsto \frac{1}{\imath \tanh \zeta}$ and eventually taking the limit $\zeta \rightarrow 0$. Keeping track of all the $\iota$ s appearing in the calculations, one arrives at (5.28) for the down-string, thus recovering the $v<1$ result exactly. In this case, there is no up-string solution.

No matter which of the three configurations we use, we can write down the expression for the Wilson loop and extract the Jet-Quenching parameter

$$
\langle W(\mathcal{C})\rangle=e^{\imath\left(S(\mathcal{C})-S_{0}\right)}=e^{-\frac{T L^{2} L^{-}}{30 \alpha^{\prime} B}} \stackrel{!}{=} e^{-\frac{1}{4 \sqrt{2}} \hat{q} L^{2} L^{-}}+\mathcal{O}\left(\frac{1}{N^{2}}\right)
$$

In each case the integral $B$ is divergent, and so the jet-quenching factor vanishes.

$$
\hat{q}=0
$$

On the non-commutativity of the limits taken As we have seen above and as was noted first in [46] the limits $\eta \rightarrow \infty$ and $\Lambda \rightarrow \infty$ do not commute. In the same paper, Liu, Rajagopal, and Wiedemann give a very nice discussion of this issue, which we shall summarize here.

Mathematics From a purely formal point of view, the first indication for noncommutativity is that one needs the Lagrangian to be imaginary in order for the expectation value to be real. This leads to

$$
\frac{\Lambda^{5}}{\cosh ^{2} \eta}<1
$$

Now regard (5.25). In going from the second line to the third, we need to assume

$$
\frac{y^{5}}{\cosh ^{2} \eta} \ll 1 \Rightarrow \frac{\Lambda^{5}}{\cosh ^{2} \eta} \ll 1 .
$$

While this is a pretty strong assumption, it is certainly satisfied if one takes the $\eta \rightarrow \infty$ limit first. This corresponds with the ansatz taken in [17] where the authors work with a light-like worldsheet in the first place.

Physics As to physics, one need to consider that different types of Wilson loops may be used to study different physical problems. On the one hand, we have jet-quenching, related to a Wilson loop which is again related to the exponential of a real quantity. This is the regime $\cosh \eta \gg \Lambda$. On the other there is the behavior of the (possibly moving) $q \bar{q}$ pair, where the Wilson loop is related to the exponential of an imaginary quantity. Here we have $\cosh \eta \ll \Lambda$. Between these two regions there is a discontinuity at $\cosh \eta \sim \Lambda$. 
The authors of [46] go on to point out that if $\cosh \eta \gg 1$ but $\cosh \eta<\Lambda$, the screening length $L_{\max }$ is given by

$$
L_{\max }=\frac{0.743}{\pi \sqrt{\cosh \eta} T} .
$$

Also, there is a size $\delta$ associated with every external quark, given by

$$
\delta \sim \frac{\sqrt{\lambda}}{M} \sim \frac{1}{\Lambda T} .
$$

$M=M(\Lambda)$ is the mass of the quark. So at the singularity, the screening length is similiar to the size of the quark

$$
\delta \sim L_{\max }
$$

Now if

$$
1 \ll \cosh \eta \ll \Lambda \quad \text { then } \quad \delta \ll L_{\max }
$$

which confirms that the string represents a quarkonium meson. If we trust the above formulas to be true in the limit $\cosh \eta \gg \Lambda$, albeit not having assumed this when defining $L_{\max }$, we realize that because of

$$
\delta \gg L_{\max }
$$

the quark is bigger than its screening length, meaning that there are no $q \bar{q}$ bound states. So there are two different regimes with different physics, depending on

$$
\cosh ^{2} \eta \lessgtr \Lambda^{5} .
$$

If we want to examine certain physics, we have to make a choice on how to take the limit.

\subsubsection{Drag Force}

While the jet-quenching method described above only uses the gauge/gravity correspondence to calculate the expectation value of a wilson line, the concept of the drag force, which was introduced in $[11,45]$, is fully based on the existence of a holographic dual. The main idea is that if one is able to describe a massive quark-antiquark pair as an open string whose both ends are attached to a probe brane at large radius, one might be able to think of a single quark as a single string stretching from the probe to the horizon. Again one uses the Nambu-Goto action in order to study the string's dynamics.

Generically the movement of the quark trough the plasma is governed by

$$
\dot{p}=-\mu p+f,
$$

where $p$ is the quarks momentum, $\mu$ a damping coefficient, and $f$ a possible external force. There are two situations of interest here. $f=0$ and $\dot{p}=0$.

In the first case, it follows that $\frac{\dot{p}}{p}=-\mu$ and therefore

$$
p(t)=e^{-\mu t} p(0) .
$$

One may extract $\mu$ numerically from a quasi normal mode analysis of a string stretching between the probe and the boundary. 
We shall however not perform the numerical analysis and instead only focus on the second case. A quark moving at a constant speed through the plasma satisfies $\dot{p}=0$. Yet as the plasma is continuously draining the quark's energy, there has to be an external force $f$ constantly repleneshing the quark's energy and momentum.

Again we place the probe brane at $\rho=\Lambda \rho_{0}$. To study a single open string hanging down to the horizon, we assume a profile of the form

$$
\tau=t \quad \sigma=\rho \quad x=x(\tau, \sigma)
$$

where in opposite to (3.26) we allow $x$ to depend on the time. The Nambu-Goto action (3.19) yields the following equations of motion

$$
0=-g_{\rho \rho} g_{x x} \partial_{\tau} \frac{\dot{x}}{\sqrt{-g}}+\partial_{\rho} \frac{-g_{t t} g_{x x} x^{\prime}}{\sqrt{-g}}
$$

where we defined

$$
\begin{aligned}
g & =g_{t t} g_{\rho \rho}+g_{t t} g_{x x} x^{\prime 2}+g_{\rho \rho} g_{x x} \dot{x}^{2}, \\
& =-\frac{\rho^{2}}{9 N^{2} \rho_{0}^{2}}-\frac{\rho^{2}}{9 N^{2} \rho_{0}^{2}} f(\rho) x^{\prime 2}+\frac{\rho^{2}}{9 N^{2} \rho_{0}^{2}} \frac{1}{f(\rho)} \dot{x}^{2},
\end{aligned}
$$

We shall now examine the properties of a specific time-dependent solution. As we will see one may extract information about the string and the quark it describes without fully solving the equations of motion.

Assume $\partial_{t} x=v$, a constant. Then the equations (5.43) and (5.42) simplify to

$$
\begin{aligned}
g & =g_{t t} g_{\rho \rho}+g_{t t} g_{x x} x^{\prime 2}+g_{\rho \rho} g_{x x} v^{2} \\
& =-\frac{\rho^{2}}{9 N^{2} \rho_{0}^{2}}-\frac{\rho^{2}}{9 N^{2} \rho_{0}^{2}} f(\rho) x^{\prime 2}+\frac{\rho^{2}}{9 N^{2} \rho_{0}^{2}} \frac{1}{f(\rho)} v^{2}
\end{aligned}
$$

and

$$
0=\partial_{\rho} \frac{-g_{t t} g_{x x} x^{\prime}}{\sqrt{-g}}
$$

as $\partial_{\tau} g=0$. This can be integrated once and solved for $x^{\prime}$ to give

$$
x^{\prime 2}=-\frac{C^{2} g_{\rho \rho}\left(g_{t t}+v^{2} g_{x x}\right)}{g_{t t} g_{x x}\left(g_{t t} g_{x x}+C^{2}\right)},
$$

where $C$ is a constant of integration.

Plugging this back into (3.22), (3.23) yields

$$
\begin{aligned}
& \frac{d E}{d t}=\pi_{t}^{\sigma}=-\frac{C v}{2 \pi \alpha^{\prime}} \\
& \frac{d P}{d t}=-\pi_{x}^{\sigma}=-\frac{C}{2 \pi \alpha^{\prime}}
\end{aligned}
$$

We want the string to reach the horizon. To see whether this is possible, we need to check if the solution is well defined in the region $\rho_{0} \leq \rho \leq \Lambda \rho_{0}$. As usual one needs to require $\sqrt{-g}, x^{2} \geq 0$. From

$$
\sqrt{-g}=-g_{t t} g_{x x} x^{\prime} C^{-1}
$$


it follows that $\sqrt{-g}$ is real if that is the case for $x^{\prime 2}$. A look at (5.46) tells us that we cannot avoid its numerator to change the sign as long as $v \neq 0$. Hence one needs to make sure that both the numerator and the denominator change sign at the same radial position $\rho_{ \pm}$. This amounts to solving

$$
g_{t t}+\left.g_{x x} v^{2}\right|_{\rho=\rho_{ \pm}}=0=g_{x x} g_{t t}+\left.C^{2}\right|_{\rho=\rho_{ \pm}}
$$

for $C$. The former equation leads to $\rho_{+}=\rho_{h}\left(1-v^{2}\right)^{1 / 5}$, from which it follows that

$$
C=\frac{v}{3 N_{c}\left(1-v^{2}\right)^{1 / 6}} .
$$

Hence the energy and momentum loss are

$$
\begin{aligned}
& \frac{d P}{d t}=-\frac{v}{6 \pi N \alpha^{\prime}\left(1-v^{2}\right)^{1 / 5}} \\
& \frac{d E}{d t}=-\frac{v^{2}}{6 \pi N \alpha^{\prime}\left(1-v^{2}\right)^{1 / 5}}
\end{aligned}
$$

Going back to (5.39), setting $\dot{p}=0$, taking (5.52) for $-f$, and making use of the relativistic relation $p=\frac{m v}{\sqrt{1-v^{2}}}$, leads to

$$
\mu m=\frac{\left(1-v^{2}\right)^{3 / 10}}{6 \pi N \alpha^{\prime}}
$$

This result has some interesting properties. As long as we consider $\alpha^{\prime}$ to be finite, the strict $N \rightarrow \infty$ limit leads to a vanishing $\mu m$. So in this case there is no radiative energy loss. This agrees nicely with the vanishing of the jet-quenching factor $\hat{q}$ studied in section 5.4.2. Furthermore (5.54) even extends that result to quarks of any non-vanishing mass. ${ }^{14}$ If we only take $N$ to be large however, equation (5.54) seems rather awkward, as the damping decreases the faster the probe moves.

Also one should not forget that we need $\alpha^{\prime}$ to be small in order to use the supergravity approximation. More precisely, as was studied in section 5.1, the 't Hooft coupling behaves as $\lambda \sim N^{5} \frac{\alpha^{\prime 2}}{\rho^{5}\left(\rho^{5}-\rho_{h}^{5}\right)}$. Thus

$$
\mu m \sim \frac{N^{3 / 2}}{\sqrt{\lambda\left(\rho^{10}-\rho^{5} \rho_{h}^{5}\right)}} .
$$

So making a definite statement about the fate of the damping coefficient $\mu$ requires a more rigorous study of the relation between the gauge- and the string theory's couplings and energy scales.

\section{Conclusions}

We have constructed a new solution (4.4) to the equations of motion of elevendimensional supergravity. As our discussion of its thermodynamical properties

\footnotetext{
${ }^{14}$ Note however that to take the limit $m \rightarrow 0$ one needs to bring the probe brane arbitrarily close to the horizon. One should assume that something should happen in this case, i.e. the brane might fall into the horizon.
} 
in section 4.2 shows there is reason to doubt that it is dual to a four-dimensional gauge theory at finite temperature, leaving us with the question what the fieldtheory dual of the background in question is. Our comparison with the four- and eleven-dimensional Schwarzschild black holes shows however that the surprising thermodynamical features are to be expected from a solution that is purely gravitational in eleven dimensions. Therefore one might expect to find a better supergravity dual upon generalizing the ansatz (4.1) such that the black hole is charged under the three-form gauge field of eleven-dimensional supergravity.

Albeit these problems we were able to exhibit some of the expected features of a gauge-dual at $T>0$, such as deconfinement and the universal ratio of shear-viscosity and entropy density. Further pathologies of our background are the negative specific heat and the vanishing parton energy loss.

As to the issue of the specific heat one should call to mind the work done by Gubser and Mitra [51, 52, 53], indicating that in fairly general settings a thermodynamic instability is leading to a dynamical one.

One might also consider the following: While our derivation of the shearviscosity to entropy ratio uses the concept of the stretched horizon introduced by Kovtun, Son, and Starinets [41], one expects to obtain the same universal result from the more standard calculation based on the evaluation of the Kuborelations. Now as the derivation of photon and dilepton production in the dual plasma [9] is quite similiar ot that of the shear-viscosity one might conjecture these quantities to behave better then the energy loss that was was discussed in this paper.

\section{Acknowledgements}

I would like to thank Gert Aarts, Adi Armoni, Carlos Hoyos, Prem Kumar, Asad Naqvi, Yiannis Papadimitriou, Jefferson Ridgway, and especially Carlos Núñez for their advice and the many discussions that led to the results reported in this paper. There are further thanks due to Carlos Núñez who gave me the original idea for this project. Also I would like to thank Gaetano Bertoldi for his continous interest in my work. Finally I am grateful to Justin Vázquez-Poritz for a discussion on the role of the different string configurations related to jet quenching and to Nestor Armesto for his guidance towards the literature on jet-quenching and phaenomenology. I am supported by a PPARC fellowship and a Ph.D. scholarship of the Studienstiftung des deutschen Volkes (German National Academic Foundation).

\section{A The bundle structure of $S^{3}$}

We examine the bundle structure of $S^{3}$, following the classic book by Nakahara [54]. The 3 -sphere can be defined as

$$
S^{3} \equiv\left\{\left.\left(z_{0}, z_{1}\right) \in \mathbb{C}^{2}|| z_{0}\right|^{2}+\left|z_{1}\right|^{2}=1\right\}
$$


In the language of [54] this is our total space. Being a manifold, we can equip it with an open covering

$$
\begin{aligned}
U_{0} & \equiv\left\{\left.\left(z_{0}, z_{1}\right) \in S^{3}|| z_{0}\right|^{2} \leq \frac{1}{2}\right\} \\
U_{1} & \equiv\left\{\left.\left(z_{0}, z_{1}\right) \in S^{3}|| z_{1}\right|^{2} \leq \frac{1}{2}\right\} \\
U_{0} \cap U_{1} & =\left\{\left(z_{0}, z_{1}\right)|| z_{0}\left|=\frac{1}{\sqrt{2}}=\right| z_{1} \mid\right\}
\end{aligned}
$$

We claim that the base space is $S^{2}$ and the fibre $S^{1} \simeq \mathcal{U}(1)$. To show this, let us first define the projection.

$$
\begin{aligned}
\pi: S^{3} & \rightarrow S^{2} \simeq \mathbb{C P}^{1} \\
\left(z_{0}, z_{1}\right) & \mapsto\left[\left(z_{0}, z_{1}\right)\right]=\left\{\lambda\left(z_{0}, z_{1}\right) \mid \lambda \in \mathbb{C} \backslash\{0\}\right\}
\end{aligned}
$$

Now on $U_{0,1}$, we know that $z_{1,0} \neq 0$ and can thus choose $\lambda=z_{1,0}^{-1}$. That means we have the following coordinates on $V_{0,1} \equiv \pi\left(U_{0,1}\right)$ :

$$
\zeta_{0,1} \equiv \frac{z_{0,1}}{z_{1,0}} \quad\left|\zeta_{0,1}\right| \leq 1
$$

There is an overlap between the two coordinate patches

$$
V_{0} \cap V_{1}=\left\{\left|\zeta_{0}\right|=1=\left|\zeta_{1}\right|\right\}
$$

on which the coordinates are related as $\zeta_{0}=\zeta_{1}^{-1}$. Our base space has thus the topology of two discs glued together along their boundaries and is therefore a two-sphere.

To confirm that the fibre is indeed $\mathcal{U}(1)$, we need to examine $\pi^{-1}$. Choose $\zeta \in S^{2}$. We shall assume w.l.o.g. $\zeta \in V_{0}$. We can somewhat lift $\zeta$ to $\mathbb{C P}^{1}$ by writing

$$
\zeta=(\zeta, 1) \simeq \lambda(\zeta, 1) \quad \lambda \in \mathbb{C} \backslash\{0\}
$$

We are now looking for points in $S^{3}$ which are projected onto this element of $\mathbb{C P}^{1}$. This is summarised by the equation

$$
\kappa\left(z_{0}, z_{1}\right)=\lambda(\zeta, 1)
$$

The $\mathbb{C}$-number $\kappa$ is redundant, leading us to

$$
\left(z_{0}, z_{1}\right)=(\lambda \zeta, \lambda) \Rightarrow|\lambda|^{2}|\zeta|^{2}+|\lambda|^{2}=1
$$

While this uniquely determines the modulus of $\lambda$, its complex phase remains fully arbitrary. We can summarize this as

$$
\pi^{-1}(\zeta) \simeq \mathcal{U}(1) .
$$

If we assume the structural group to be $\mathcal{U}(1)$, it is obvious that there is a well defined left action on the fibre.

To define the local trivilisations, we shall use the open covering $V_{i}$ of $S^{2}$ that we defined previously. Thanks to our work in the previous paragraphs, it is no work at all to write an explicit expression.

$$
\begin{aligned}
\Phi_{0}: V_{0} \times \mathcal{U}(1) & \rightarrow \pi^{-1}\left(V_{0}\right)=U_{0} \\
(\zeta, \phi) & \mapsto\left(r e^{\imath \phi} \zeta, r e^{\imath \phi}\right)
\end{aligned}
$$


with

$$
r=|\lambda|=\sqrt{\frac{1}{1+|\zeta|^{2}}}
$$

One can check that

$$
\pi\left(r e^{\imath \phi} \zeta, r e^{\imath \phi}\right)=\lambda\left(r e^{\imath \phi} \zeta, r e^{\imath \phi}\right)=(\zeta, 1)=\zeta .
$$

A virtually identical definition holds for $V_{1}$.

$$
\begin{aligned}
\Phi_{1}: V_{1} \times \mathcal{U}(1) & \rightarrow \pi^{-1}\left(V_{1}\right)=U_{1} \\
(\zeta, \phi) & \mapsto\left(r e^{\imath \phi}, r e^{\imath \phi} \zeta\right)
\end{aligned}
$$

Finally, we check the transition functions. Assume $\zeta \in V_{0} \cap V_{1}$; it follows that $\zeta=e^{\imath \theta}$.

$$
\begin{aligned}
t_{01, \zeta}(\phi) & =\Phi_{1}^{-1}\left(r e^{\imath \phi} \zeta, r e^{\imath \phi}\right) \\
& =\left(\zeta^{-1}, \phi+\theta\right) \in V_{1} \times \mathcal{U}(1)
\end{aligned}
$$

This shows that the transition function is a simple shift in the fibre and thus certainly a diffeomorphism. Note that in going to the last line, we had to acknowledge that when going from $V_{0}$ to $V_{1}$ coordinates, we have to invert the element.

\section{References}

[1] J. M. Maldacena, "The large $\mathrm{N}$ limit of superconformal field theories and supergravity," Adv. Theor. Math. Phys. 2, 231 (1998) [Int. J. Theor. Phys. 38, 1113 (1999)] [arXiv:hep-th/9711200].

[2] E. Witten, "Anti-de Sitter space and holography," Adv. Theor. Math. Phys. 2, 253 (1998) [arXiv:hep-th/9802150].

[3] S. S. Gubser, I. R. Klebanov and A. M. Polyakov, "Gauge theory correlators from non-critical string theory," Phys. Lett. B 428, 105 (1998) [arXiv:hepth/9802109].

[4] I. Arsene et al. [BRAHMS Collaboration], "Quark gluon plasma and color glass condensate at RHIC? The perspective from the BRAHMS experiment," Nucl. Phys. A 757, 1 (2005) [arXiv:nucl-ex/0410020].

[5] B. B. Back et al., "The PHOBOS perspective on discoveries at RHIC," Nucl. Phys. A 757, 28 (2005) [arXiv:nucl-ex/0410022].

[6] J. Adams et al. [STAR Collaboration], "Experimental and theoretical challenges in the search for the quark gluon plasma: The STAR collaboration's critical assessment of the evidence from RHIC collisions," Nucl. Phys. A 757, 102 (2005) [arXiv:nucl-ex/0501009].

[7] K. Adcox et al. [PHENIX Collaboration], "Formation of dense partonic matter in relativistic nucleus nucleus collisions at RHIC: Experimental evaluation by the PHENIX collaboration," Nucl. Phys. A 757, 184 (2005) [arXiv:nucl-ex/0410003]. 
[8] G. Policastro, D. T. Son and A. O. Starinets, "The shear viscosity of strongly coupled $\mathrm{N}=4$ supersymmetric Yang-Mills plasma," Phys. Rev. Lett. 87, 081601 (2001) [arXiv:hep-th/0104066].

[9] S. Caron-Huot, P. Kovtun, G. D. Moore, A. Starinets and L. G. Yaffe, "Photon and dilepton production in supersymmetric Yang-Mills plasma," JHEP 0612, 015 (2006) [arXiv:hep-th/0607237].

[10] H. Liu, K. Rajagopal and U. A. Wiedemann, "Calculating the jet quenching parameter from AdS/CFT," Phys. Rev. Lett. 97, 182301 (2006) [arXiv:hep$\mathrm{ph} / 0605178]$.

[11] C. P. Herzog, A. Karch, P. Kovtun, C. Kozcaz and L. G. Yaffe, "Energy loss of a heavy quark moving through $\mathrm{N}=4$ supersymmetric Yang-Mills plasma," JHEP 0607, 013 (2006) [arXiv:hep-th/0605158].

[12] D. Mateos, "String Theory and Quantum Chromodynamics," arXiv:0709.1523 [hep-th].

[13] K. Yagi, T. Hatsuda and Y. Miake, "Quark-Gluon Plasma: From Big Bang To Little Bang," Cambridge, UK: Univ. Pr. (2005) $446 p$

[14] P. Jacobs and X. N. Wang, "Matter in extremis: Ultrarelativistic nuclear collisions at RHIC," Prog. Part. Nucl. Phys. 54, 443 (2005) [arXiv:hep$\mathrm{ph} / 0405125]$.

[15] A. Buchel, "On jet quenching parameters in strongly coupled nonconformal gauge theories," Phys. Rev. D 74, 046006 (2006) [arXiv:hepth/0605178].

[16] G. Bertoldi, F. Bigazzi, A. L. Cotrone and J. D. Edelstein, "Holography and Unquenched Quark-Gluon Plasmas," Phys. Rev. D 76, 065007 (2007) [arXiv:hep-th/0702225].

[17] N. Armesto, J. D. Edelstein and J. Mas, "Jet quenching at finite 't Hooft coupling and chemical potential from AdS/CFT," JHEP 0609, 039 (2006) [arXiv:hep-ph/0606245].

[18] A. L. Cotrone, J. M. Pons and P. Talavera, "Notes on a SQCD-like plasma dual and holographic renormalization," arXiv:0706.2766 [hep-th].

[19] S. D. Avramis and K. Sfetsos, "Supergravity and the jet quenching parameter in the presence of R-charge densities," JHEP 0701, 065 (2007) [arXiv:hep-th/0606190].

[20] E. Witten, "Topological Quantum Field Theory," Commun. Math. Phys. 117, 353 (1988).

[21] R. Gopakumar and C. Vafa, "On the gauge theory/geometry correspondence," Adv. Theor. Math. Phys. 3, 1415 (1999) [arXiv:hep-th/9811131].

[22] C. Vafa, "Superstrings and topological strings at large N," J. Math. Phys. 42, 2798 (2001) [arXiv:hep-th/0008142]. 
[23] A. Brandhuber, J. Gomis, S. S. Gubser and S. Gukov, "Gauge theory at large N and new G(2) holonomy metrics," Nucl. Phys. B 611, 179 (2001) [arXiv:hep-th/0106034].

[24] M. Atiyah, J. M. Maldacena and C. Vafa, "An M-theory flop as a large N duality," J. Math. Phys. 42, 3209 (2001) [arXiv:hep-th/0011256].

[25] J. D. Edelstein and C. Nunez, "D6 branes and M-theory geometrical transitions from gauged supergravity," JHEP 0104, 028 (2001) [arXiv:hepth/0103167].

[26] N. Itzhaki, J. M. Maldacena, J. Sonnenschein and S. Yankielowicz, "Supergravity and the large N limit of theories with sixteen supercharges," Phys. Rev. D 58, 046004 (1998) [arXiv:hep-th/9802042].

[27] P. Candelas and X. C. de la Ossa, "Comments on Conifolds," Nucl. Phys. B 342, 246 (1990).

[28] E. Kiritsis, "String theory in a nutshell," Princeton, USA: Univ. Pr. (2007) $588 p$

[29] K. Becker, M. Becker and J. H. Schwarz, "String theory and M-theory: A modern introduction," Cambridge, UK: Cambridge Univ. Pr. (2007) 739 p

[30] J. D. Edelstein and R. Portugues, "Gauge / string duality in confining theories," Fortsch. Phys. 54, 525 (2006) [arXiv:hep-th/0602021].

[31] M. Bertolini and P. Merlatti, "A note on the dual of $\mathrm{N}=1$ super Yang-Mills theory," Phys. Lett. B 556, 80 (2003) [arXiv:hep-th/0211142].

[32] P. Di Vecchia, A. Lerda and P. Merlatti, " $N=1$ and $N=2$ super Yang-Mills theories from wrapped branes," Nucl. Phys. B 646, 43 (2002) [arXiv:hepth/0205204].

[33] J. M. Maldacena, "Wilson loops in large N field theories," Phys. Rev. Lett. 80, 4859 (1998) [arXiv:hep-th/9803002].

[34] S. J. Rey and J. T. Yee, "Macroscopic strings as heavy quarks in large N gauge theory and anti-de Sitter supergravity," Eur. Phys. J. C 22, 379 (2001) [arXiv:hep-th/9803001].

[35] N. Drukker, D. J. Gross and H. Ooguri, "Wilson loops and minimal surfaces," Phys. Rev. D 60, 125006 (1999) [arXiv:hep-th/9904191].

[36] P. C. Argyres, M. Edalati and J. F. Vazquez-Poritz, "Spacelike strings and jet quenching from a Wilson loop," JHEP 0704, 049 (2007) [arXiv:hepth/0612157].

[37] J. Sonnenschein, "What does the string / gauge correspondence teach us about Wilson loops?," arXiv:hep-th/0003032.

[38] K. G. Wilson, "Confinement of quarks," Phys. Rev. D 10, 2445 (1974).

[39] Y. Kinar, E. Schreiber and J. Sonnenschein, "Q anti-Q potential from strings in curved spacetime: Classical results," Nucl. Phys. B 566, 103 (2000) [arXiv:hep-th/9811192]. 
[40] E. Witten, "Anti-de Sitter space, thermal phase transition, and confinement in gauge theories," Adv. Theor. Math. Phys. 2, 505 (1998) [arXiv:hepth/9803131].

[41] P. Kovtun, D. T. Son and A. O. Starinets, "Holography and hydrodynamics: Diffusion on stretched horizons," JHEP 0310, 064 (2003) [arXiv:hepth/0309213].

[42] P. Kovtun, D. T. Son and A. O. Starinets, "Viscosity in strongly interacting quantum field theories from black hole physics," Phys. Rev. Lett. 94, 111601 (2005) [arXiv:hep-th/0405231].

[43] A. Buchel and J. T. Liu, "Universality of the shear viscosity in supergravity," Phys. Rev. Lett. 93, 090602 (2004) [arXiv:hep-th/0311175].

[44] J. Adams et al. [STAR Collaboration], "Evidence from d + Au measurements for final-state suppression of high $\mathrm{p}(\mathrm{T})$ hadrons in $\mathrm{Au}+\mathrm{Au}$ collisions at RHIC," Phys. Rev. Lett. 91, 072304 (2003) [arXiv:nucl-ex/0306024].

[45] S. S. Gubser, "Drag force in AdS/CFT," Phys. Rev. D 74, 126005 (2006) [arXiv:hep-th/0605182].

[46] H. Liu, K. Rajagopal and U. A. Wiedemann, "Wilson loops in heavy ion collisions and their calculation in AdS/CFT," JHEP 0703, 066 (2007) [arXiv:hep-ph/0612168].

[47] P. C. Argyres, M. Edalati and J. F. Vazquez-Poritz, "No-drag string configurations for steadily moving quark-antiquark pairs in a thermal bath," JHEP 0701, 105 (2007) [arXiv:hep-th/0608118].

[48] P. C. Argyres, M. Edalati and J. F. Vazquez-Poritz, "Spacelike strings and jet quenching from a Wilson loop," JHEP 0704, 049 (2007) [arXiv:hepth/0612157].

[49] P. C. Argyres, M. Edalati and J. F. Vazquez-Poritz, "Lightlike Wilson loops from AdS/CFT," JHEP 0803, 071 (2008) [arXiv:0801.4594 [hep-th]].

[50] U. A. Wiedemann, "Gluon radiation off hard quarks in a nuclear environment: Opacity expansion," Nucl. Phys. B 588, 303 (2000) [arXiv:hep$\mathrm{ph} / 0005129]$.

[51] S. S. Gubser and I. Mitra, "Instability of charged black holes in anti-de Sitter space," arXiv:hep-th/0009126.

[52] S. S. Gubser and I. Mitra, "The evolution of unstable black holes in anti-de Sitter space," JHEP 0108, 018 (2001) [arXiv:hep-th/0011127].

[53] J. J. Friess, S. S. Gubser and I. Mitra, "Counter-examples to the correlated stability conjecture," Phys. Rev. D 72, 104019 (2005) [arXiv:hepth/0508220].

[54] M. Nakahara, "Geometry, topology and physics," Boca Raton, USA: Taylor 8 Francis (2003) $573 p$ 